\title{
Software-defined Networking Based DDoS Defense
} Mechanisms

This paper was downloaded from TechRxiv (https://www.techrxiv.org).

\section{LICENSE}

CC BY-NC-SA 4.0

SUBMISSION DATE / POSTED DATE

$26-07-2020$ / 28-07-2020

CITATION

Swami, Rochak; Dave, Mayank; Ranga, Virender (2020): Software-defined Networking Based DDoS Defense Mechanisms. TechRxiv. Preprint. https://doi.org/10.36227/techrxiv.12720482.v1

DOI

10.36227/techrxiv.12720482.v1 


\title{
Software Defined Networking Based DDoS Defense Mechanisms
}

\author{
ROCHAK SWAMI, MAYANK DAVE, and VIRENDER RANGA, National Institute of
} Technology, India

Distributed Denial of Service attack (DDoS) is recognized to be one of the catastrophic attacks against various digital communication entities. Software-defined networking (SDN) is an emerging technology for computer networks that uses open protocols for controlling switches and routers placed at the network edges by using specialized open programmable interfaces. In this paper, a detailed study on DDoS threats prevalent in SDN is presented. Firstly, SDN features are examined from the perspective of security, and then, a discussion on assessment of SDN security features is done. Further, two viewpoints towards protecting the networks against DDoS attacks are elaborated In the first view, SDN utilizes its abilities to secure the conventional networks. In the second view, SDN may become a victim of the threats itself because of the centralized control mechanism. The main focus of this research work is towards discovering critical security implications in SDN while reviewing the current ongoing research studies. By emphasizing the available state of the art techniques, an extensive review towards the advancement of the SDN security is provided to the researchers and IT communities.

CCS Concepts: • Security and privacy $\rightarrow$ Intrusion detection systems; Denial-of-service attacks; Security services;

Additional Key Words and Phrases: Software defined networking, Distributed Denial of Service, Control plane, Data plane

\section{ACM Reference Format:}

Rochak Swami, Mayank Dave, and Virender Ranga. 2018. Software Defined Networking Based DDoS Defense Mechanisms. ACM Comput. Surv. 1, 1, Article 1 (January 2018), 37 pages. https: //doi.org/10.1145/3301614

\section{INTRODUCTION}

Networking has become an essential part of our lives to share information and resources in digital information technology. It is a process of communicating with other devices digitally. However, the current IP networks are not flexible and have a static architecture in which reconfiguration of the new policies and rules are difficult. The reason is that there is a strong coupling of control and data plane, which means that the controlling and routing policies are embedded into the data forwarding devices/hardware. This property makes it more difficult to manage the network and its protocols dynamically. Whenever there is a need to update any existing policy or to add a new functionality, configuration of all the affected

Authors' address: Rochak Swami; Mayank Dave; Virender Ranga, National Institute of Technology, Department of Computer Engineering, Kuruskhetra, Haryana, 136119, India, rochakswami123@gmail.com, mdave@nitkkr.ac.in, virender.ranga@nitkkr.ac.in.

Permission to make digital or hard copies of all or part of this work for personal or classroom use is granted without fee provided that copies are not made or distributed for profit or commercial advantage and that copies bear this notice and the full citation on the first page. Copyrights for components of this work owned by others than ACM must be honored. Abstracting with credit is permitted. To copy otherwise, or republish, to post on servers or to redistribute to lists, requires prior specific permission and/or a fee. Request permissions from permissions@acm.org.

(C) 2018 Association for Computing Machinery.

0360-0300/2018/1-ART1 \$15.00

https://doi.org/10.1145/3301614

ACM Comput. Surv., Vol. 1, No. 1, Article 1. Publication date: January 2018. 
devices are modified. This process is time consuming and increases overall cost of the process. To produce more optimized and better results, there is a need to make networks easily adaptable and reconfigurable. Moreover, in case of cloud computing, service providers need to fulfill the demands of business customers. To achieve this goal, network needs to be more programmable and agile according to the time bound requirements for new applications viz. network virtualization [1]. To overcome the limitations of current networks, a new technology named Software-defined networking (SDN) has been developed as a revolutionary paradigm to give the next-generation architecture for the Future Internet [2]. SDN ensures the desired flexibility for networks. SDN first came into existence by OpenFlow at Stanford University. Open Networking Foundation (ONF) gave a push towards adoption of SDN by developing the OpenFlow protocol $[3,4]$. OpenFlow is designed as one of the first SDN standards. OpenFlow protocol is responsible for intercommunication between the two planes of SDN (control and data). The OpenFlow was proposed in 2008 to provide the flexibility and programmability. The ONF is being used by well known organizations including Deutsche Telekom, Verizon and Yahoo! since 2011 [5]. A development timeline of SDN techniques throughout the years is shown in Fig. 1.

SDN has gained attention of the researchers for the future generation networks. It has been adopted for its virtualized and flexible behavior by both academic and industry communities. The main characteristic of SDN is detachment of control and data plane [6]. The controller manages and controls all the forwarding devices (router, switches) residing in the data plane. This makes forwarding devices no longer smarter. They act as normal forwarding devices. This unique property of SDN makes it different from traditional networking technologies, which combine both the planes (control and data plane) tightly with each other. Using this idea, complete functionality is managed by software programming without any modification in the existing network topologies [7]. In SDN if any rule/policy updates are needed according to user's requirements, these changes are implemented only in the control plane thereby reducing the cost of this process. The centralized infrastructure of SDN can provide an efficient use of the resources and improvement of the network performance. SDN makes the network more programmable and innovative. SDN is replacing the traditional networking technologies because of many advantages: it provides a complete view of the network, gives logically centralized control, programmability, simplification of network management, easy reconfiguration, and open programmable interfaces. Advantages of SDN technologies are shown in Fig. 3. With the growth of this networking paradigm, various threats against SDN have also grown in order to disrupt its normal operation.

While discussing network security, CIA triad [8] is designed as one of the most important models for security policies. The CIA triad (Confidentiality, Integrity, Availability) is a benchmark model for securing the network [9]. There are many threats that attempt to damage the network's services and resources. Denial of Service (DoS) attacks [10] are considered among the most destructive cyber attacks on the network. DDoS attack exploits one of the components of the CIA model i.e., availability. DDoS attack stops legitimate users from availing internet services or resources by making superfluous requests to the systems i.e., servers and devices [11]. This huge amount of traffic is produced from multiple sources. This makes it very difficult to handle the attack situation. The scenario of DDoS or DoS is quite similar to a group of customers outside the door of a shop, trying to enter, and making it difficult for benign customers to enter [12]. On March 5, 2018, Github was victimized by one of the largest DDoS attacks that peaked about 1.7 Tbps [13]. Due to the architectural design , SDNis vulnerable to DDoS attacks from many view points. SDN has a central view of the network topology provided by the controller. However, this characteristic makes it vulnerable 
to several threats. There are many possibilities for an attacker to make modifications to the whole SDN network functioning just by changing the controller. We can analyze the security issues of SDN in two aspects. One aspect is security by SDN and another aspect is security for SDN. SDN has many capabilities for defending against the DDoS attacks that make it useful for protecting many different types of networking technologies. On the other hand, SDN attracts several attacks due to its design features such as detachment of control and data plane.

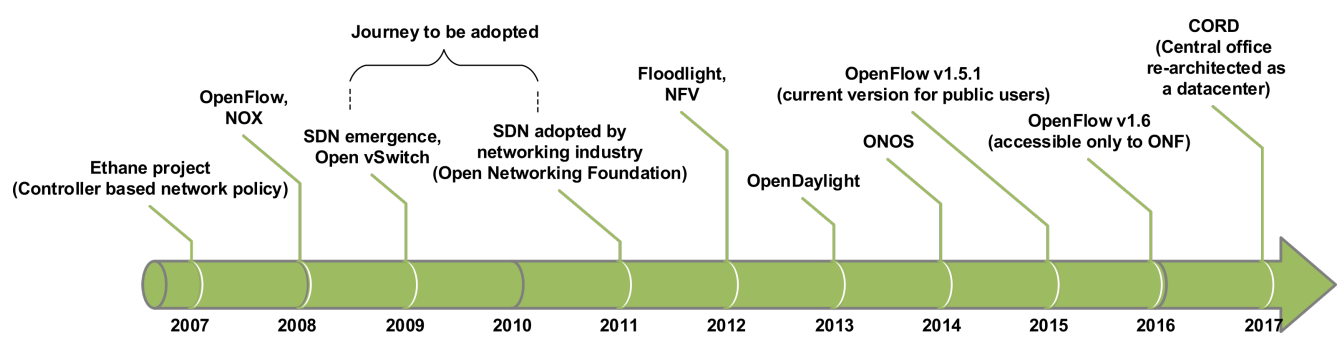

Fig. 1. Development trends in SDN

\subsection{SDN Architecture}

SDN detaches the control and forwarding (data) logic. SDN architecture is represented in the form of layers as illustrated in Fig. 2. The architecture is separated into three layers: application, control, and data forwarding. The processing starts when a packet arrives at data forwarding layer that handles the packets and if required, gives it to the control layer. Control layer may require various applications having different functionalities. These layers of SDN architecture are described below:

(1) Data forwarding layer: This layer contains different switches and routers. They are connected with each other through a wired or wireless channel. An SDN switch is simply used to forward the packets on the basis of controller's instruction. Each switch has a flow table that contains the entries of packets to make forwarding decisions. Each entry in the flow table has three parts: rule, action, and counter. The rule specifies the field values of the packet header. Whenever the switch receives a new packet, it checks the flow table to find the rule. The field values if get matched, value of the counter increases, and the respective action is taken by the switch. Similarly, if the field values do not match, switch informs the controller. The controller takes an appropriate action i.e., forwarding the packet, dropping or adding new rules to the switches.

(2) Control layer: It contains a single or multiple controllers. The complex control logic is implemented in the controller and known as "brain of SDN". The control layer controls all the switches and manages the whole network. The SDN controller and switches communicate through a standard southbound API (OpenFlow). It provides a full view of the network. When multiple controllers are used, they are connected with each other through an interface known as east-west bound API. This interface makes them sharing the essential information with each other. In the multi-controller environment, each controller handles a group of switches.

(3) Application layer: This layer contains different applications required for several business concerns and necessities. An application is a software program that is deployed over the controller. SDN applications communicate with the controller using a northbound interface (REST) according to their network requirements. Some of the required applications 


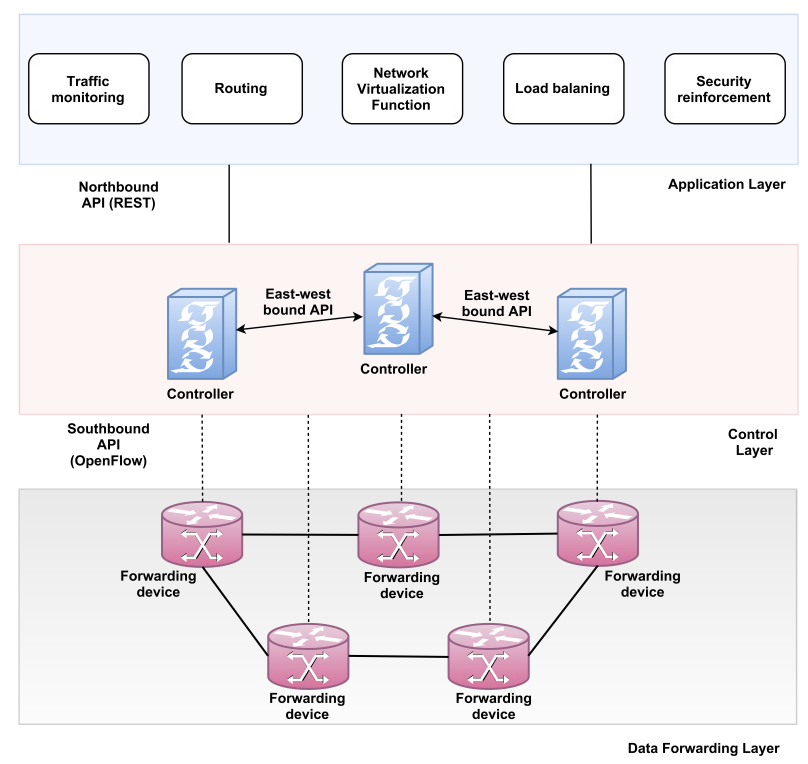

Fig. 2. SDN Architecture

are such as traffic monitoring, network virtualization, security reinforcement, load balancing, mobility management, and others. The control layer presents an abstracted view of all the physical elements to the application layer. The applications make logics for decision making used in the control layer. On the basis of this logic, data plane devices working is defined.

Some interfaces are required to provide communication between different layers in SDN. Southbound interfaces allow the communication of data plane and control plane. "Network Configuration Protocol (NetConf)" [14] and "OpenFlow" [3] are two standard southbound interfaces used in SDN implementations. Northbound interface facilitates the communication of controller and the SDN applications. Eastbound interface enables SDN to interconnect with conventional IP networks. Westbound interface allows the necessary information sharing between the controllers of different SDN domains. There are no standard northbound and east-westbound interfaces available yet.

\subsection{Security features of SDN}

SDN has several characteristics related to its architecture and design. These design characteristics make it different from conventional network architectures. We will throw light on its design features in context of security. SDN features are helpful in securing the networks with more flexibility in a fast and efficient manner. However, SDN itself may become vulnerable itself to security threats due to some defects in its design. Therefore, SDN design features can be described in two aspects. One aspect is features that make SDN resolver to DDoS threats and the other aspect is features that can make it vulnerable itself.

1.2.1 Features making SDN resolver to DDoS. SDN provides many advantageous features for dealing with DDoS: 
(1) Centralized monitoring of anomalous traffic: Controller has the complete information of the network. Therefore, all the anomalous activities going on in the network are observed by it.

(2) Programmable configuration: One of the important advantages of SDN is its programmability. Whenever any malicious behavior is detected in the network, new programs are configured immediately to deal with the anomalies.

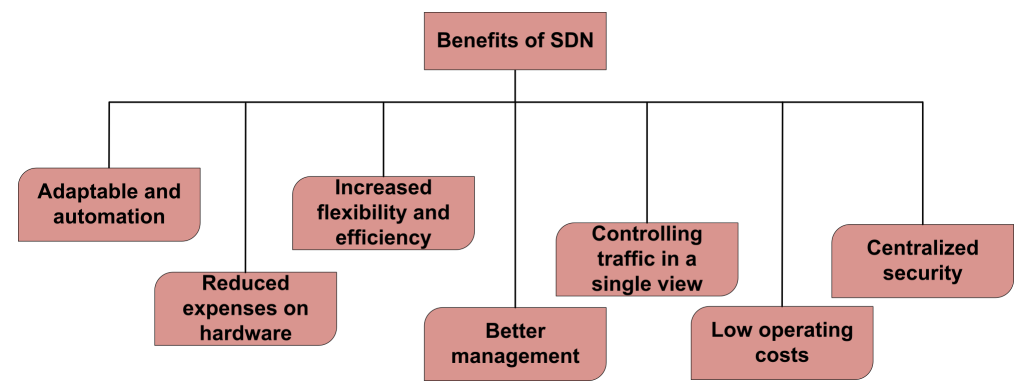

Fig. 3. Benefits of SDN technology

1.2.2 Features making SDN vulnerable to DDoS. SDN has some design issues that make it vulnerable to various security threats. The security faults in SDN design and its impact are discussed as given below.

(1) Limited TCAM: In SDN, OpenFlow switch maintains the flow rules for new incoming packets in its flow table. The switches utilize a "content addressable memory" known as TCAM (' $\mathrm{T}$ ' for ternary) [15] to reserve flow rules. It is a unique type of memory used for high speed searching applications. However, the SDN switches have the limited space of memory in its flow tables. For instance, the Pronto-Pica8 3290 switch can store only 2000 rules [16]. The limitation of the flow table memory can make the SDN sensitive to DDoS attacks.

(2) Single point of failure and cascading failure of controller: SDN controller is a prime target of the attackers. It is a centralized entity that may suffer from single point of failure. Although, the controller controls the entire network, its crashing can downgrade the network performance, availability, and integrity of the network. A single SDN controller cannot be efficient in handling the large network traffic. In that case, deploying multiple controllers in different network domains can handle the situation. There may be an issue of authenticity, consistency, and scalability of different privacy rules in each domain's controller. This may cause more than one controller getting failed in cascading manner.

(3) Decoupling of control and data plane: In SDN architecture, decoupling of these two planes makes it vulnerable to various security threats. These planes communicate using a standard protocol (OpenFlow). An attacker can disturb this communication of information by implementing DoS, saturation attack, man-in-the-middle attacks etc. to choke switch-controller channel bandwidth.

(4) Dumb switches: SDN switches are simple forwarding devices and considered as dumb [17]. They rely on the controller for taking an appropriate action to forward packets. This property of OpenFlow switches may reduce the performance of controller and control plane bandwidth because of a large amount of traffic. 


\section{DISTRIBUTED DENIAL OF SERVICE ATTACKS}

DDoS aims to disrupt ongoing operations by overwhelming network devices with connection requests for a certain time period. This flooded traffic of requests forces the target systems to slow down, crash or shut down. The DDoS attacks keep the systems busy with unusual requests by denying the services to the legitimate customers. The main reason behind DDoS attacks is that most of the networking architectures have some resource constraints. These attacks mainly deplete resources like bandwidth, memory storage and processing power. All the DDoS attacks are not same. They can target victims for different purposes. There are many DDoS attacks that are rapidly growing in the field of internet. Common types of DDoS attacks are illustrated in Fig 4. According to Arbor networks, 65\% of the total reported DDoS attacks are volumetric in general [18]. The volumetric attack sends a huge amount of data packets to the target network in order to overload its bandwidth. The protocol exploitation/state exhaustion attacks exploit the network protocols to attack on the target system, make up about $20 \%$ of the reported DDoS attacks in 2014. These attacks exploit the standard application protocols by attacking the online services e.g. web servers. These are the most challenging attacks that need to be identified and mitigated for efficient running of the network operations. The most common types of application layers attack are HTTP flood and SMTP flood. A detailed discussion on DDoS types is done in [19].

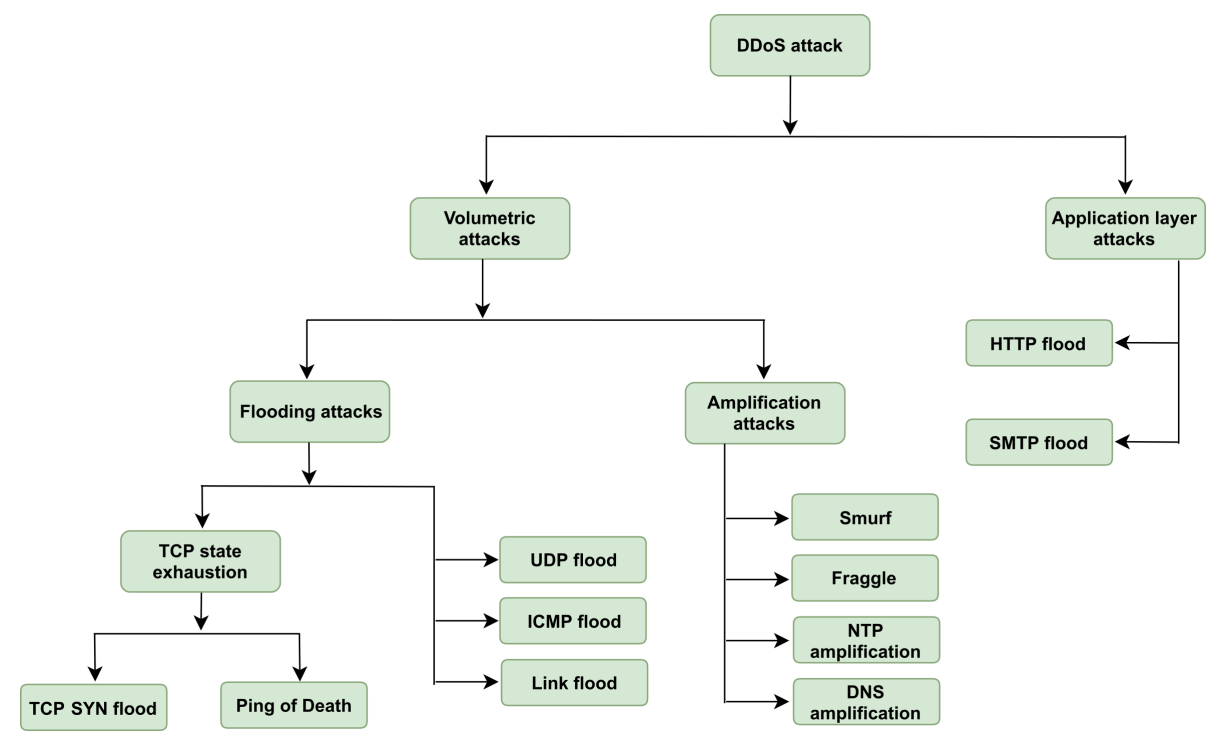

Fig. 4. Taxonomy of DDoS attacks

Slow DDoS. One more important type of DoS/DDoS attacks is slow DDoS or low rate attacks. Most common slow attacks are application layer based such as HTTP, FTP, SMTP, and IMAP. The slow DDoS attacks are very hard to detect because traffic generated by these attacks behave as legitimate traffic. These attacks utilize very less bandwidth and resources. With a small consumption of resources, they can create a large destruction. Some HTTP based slow attacks [20] are: 
(1) Slow HTTP header (Slowloris): In this attack, a header is divided into different packets and these partial headers are sent to server by attacker at a very low rate to make the service unavailable [21].

(2) Slow HTTP POST attack (RUDY): In this attack, body of POST message is divided into several packets and sent to the server at low rate.

(3) Slow read attack: Attacker sends normal HTTP request messages to the server and sees the reply very late from the server.

DDoS attacks target the network and server's resources that are listed below:

(1) Bandwidth: Flooding/volume based attacks consume all the bandwidth in the network. They do not allow the legitimate requests to reach to the server by creating exhaustion on the channel.

(2) Memory: Protocol based attacks such as SYN attack makes TCP connection open all the time that makes the buffer overflow. It consumes the TCP connection table's buffer or memory completely.

(3) $C P U$ : Application layer based attacks target on the web servers. This makes service unavailable for the legitimate users by exhausting processing power of CPU or server and server gets crashed.

Effect of $D D o S$ on $S D N$ planes. In data plane, switch has a limited size of flow tables. Due to DDoS attacks, a large volume of packets is transferred to the switches. This is called as flow rule flooding, which exhausts the flow table's memory. The attacker is motivated to send this flood to the controller for saturating the switch-controller bandwidth. One consequence of bandwidth based attack is large packet drop. The SDN switch is not able to take any decision in case of an unmatched entry and therefore sends the packet to the controller. This flooding of unmatched packets at controller consumes controller's resources (memory and $\mathrm{CPU}$ ) degrading the performance of entire system resulting in increase in response time and communication overhead.

\section{TARGET POINTS OF SECURITY THREATS IN SDN NETWORK}

The SDN architecture is divided into three layers. All the layers can be targeted by various security threats. However, the controller and control plane bandwidth are the most sensitive target points for the DDoS attacks in particular to SDN. The possible attacking targets of security threats in SDN Network are shown in Fig. 5.

(1) SDN switch: SDN switches are used for data forwarding and processing of new incoming packets. They have a very limited size of flow tables. This is a big concern for the security purpose.

(2) Links between SDN switches: The flow packets are transferred for forwarding from one switch to another switch. Most of the transferred packets are not encoded and may contain sensitive information. These packets can be intercepted by the attackers easily, especially when the links between switches are wireless.

(3) SDN controller: As the controller ("brain of SDN network") performs crucial activities for SDN, any abnormality in it can paralyze the whole network. The complete functionality of network depends on the controller. With this fact, it is the most attractive target for the attackers. It may suffer from a single point of failure in case the network has only one controller.

(4) Link between controller and switch: In case a packet arrives at switch and switch is unable to handle it, then the packet is forwarded to the controller for further processing. Consequently new packet forwarding rules are appended to the flow table of 


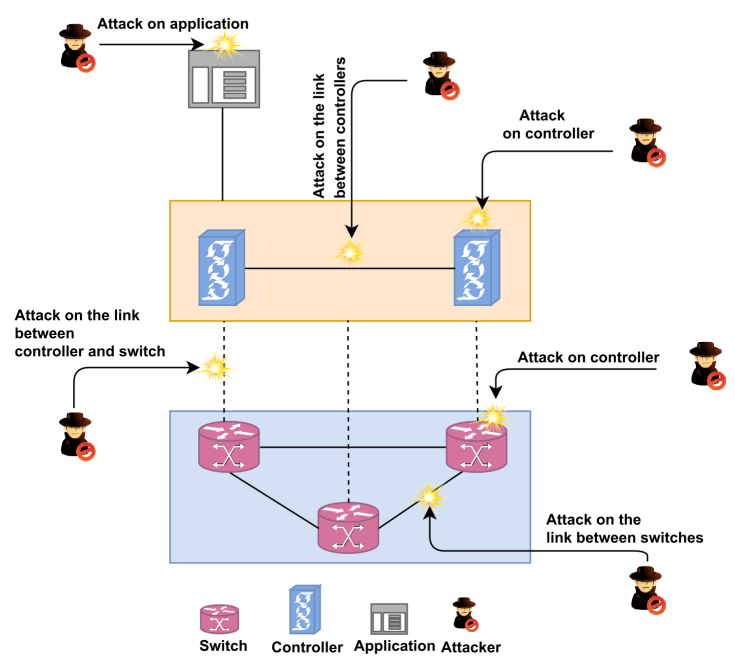

Fig. 5. Attacking Points

the switches. The rules contained in a packet are sent through the southbound interface to the switch. These data packet can be interfered by an attacker on the southbound interface that results in addition of some malicious rules or alteration of the existing rules. Placement of these fake rules in the switch table leads to misdirection of the packets.

(5) Links between two controllers: In the multi-controller based scenario, the communication is shared between the controllers through east-west bound APIs. The packets between the controllers can be obstructed by an attacker to gain essential information for compromising the controllers. Thus, the communication between controllers should be secure and authentic. The distributed controllers may also suffer from cascading failures because of flooded requests.

(6) Applications: The applications such as routing, traffic monitoring, and virtualization are implemented on the SDN control layer. Most of the applications are established by third parties that do not care for the security requirements. The applications may suffer from the unauthorized access. When the functions of the controller are called via northbound API, the malicious entities can be injected into the controllers. Hence, the SDN based applications can become the easiest target point for blocking service of controllers.

\subsection{Potential SDN specific DDoS threats}

Controller overloading and data-to-control bandwidth congestion are the most concerning DDoS/DoS threats in SDN. Some major threats are discussed here.

(1) Switch overloading and flow table overflow: DoS/DDoS attacks generate a large amount of malicious packets that are flooded into a switch. For these malicious packets, the switch will not find a corresponding entry in the flow table. It makes entries for all the unmatched requests in the buffer and sends them to the controller continuously because of unmatched rules. However, a switch has a limited size of TCAM, so it may 
not be possible to process all the incoming packets. The continued flow of requests will overflow the flow table memory and therefore, legitimate requests will have to suffer.

(2) Controller resource saturation: The controller is a heart of SDN network that controls and manages the complete functionality of the network. Hence, the compromised controller hinders the overall network performance. Controller resources such as CPU, memory will be exhausted by processing the flooded requests of the DDoS attacks. When a controller is overloaded, it can not process the new incoming flows. It degrades the performance of the complete network because legitimate requests are not handled timely.

(3) Switch-to-controller bandwidth congestion: On table-miss event due to new incoming packets, two actions are performed. First, incoming packets are buffered in the flow table of the switch. Second, an OpenFlow request is created that contains an ID and partial information of the packet header. When this buffer gets full, the complete packet is forwarded to the controller that leads to the OpenFlow channel congestion. Consequently, many packets collide with the southbound interface. In such situation, normal users face the unavailability of the services.

Defense mechanisms against DDoS. SDN security has become a hot topic for researchers in recent years. SDN helps to mitigate attacks including DDoS, IP spoofing, malware in conventional networks. However, an attacker may successfully carry out DDoS attack on SDN itself. Accordingly, the defense solutions are classified on the basis of design features of SDN as shown in Fig. 6. The first category is the set of solutions against DDoS attacks offered by SDN. The second category is the defense solutions for securing SDN from DDoS attacks.

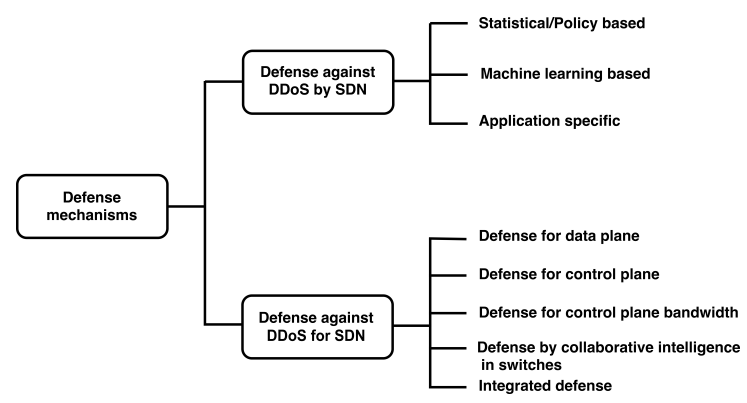

Fig. 6. Defense mechanisms for detection and mitigation of DDoS

\section{DEFENSE MECHANISMS OFFERED BY SDN AGAINST DDOS}

SDN has attracted researchers world-wide due to its effective characteristics for solving and providing new security mechanisms. With the recent upgradation of SDN, it has been a beneficial aspect for the security perspective in the traditional networks. The global view and programmability are the key features to control the impacts of DDoS attacks. Various detection and mitigation mechanisms have been described in this section. As per the utilized detection algorithms, all the existing defense mechanisms can be classified into statistical based, machine learning (ML) based, and application specific mechanisms. 


\subsection{Statistical/Policy based defense mechanisms}

In this section of defense mechanisms, statistical and policy based detection techniques are discussed. The analysis is formulated on the basis of the behavior and properties of the network flow. A statistical analysis involves collecting and exploring the data samples to identify the malicious traffic. A comparison of defense mechanisms is shown in Table 1. In this type of analysis, statistical inference test is applied on the network traffic, and if the data cannot be fitted on some statistical models, then that is classified as malicious data. In [22], the authors presented that statistical based algorithms such as entropy and chi-square for detecting the DDoS attacks accurately. Some commonly used statistical techniques in SDN are based on adaptive correlation analysis, standard deviation, probability, and entropy measurements. For detecting and mitigating DDoS attacks, policies/rules can also be defined. Many defense techniques have adopted the policy based solutions in SDN. These policy based detection techniques involve the implementation of some defined policies or rules on network traffic flows. Thus, if the traffic flows follow these policies, they are considered as legitimate flows, otherwise declared as malicious flows. Policy based solutions play the role of firewall and allow only authentic packet flows to pass through it.

Sattar et al. [23] proposed an SDN based approach named "Adaptive Bubble Burst (ABB)" to mitigate the DDoS attacks. ABB enhances availability of targeted resources under DDoS attack. ABB replicates the various copies of resource and spreads the attack over these copies. This spreading decreases the effect of the attack on actual resources. The proposed approach does not provide an idea to discover the DDoS attack. One advantage of ABB is that it has no requirement of any software update for the client-server sides. ABB as a mitigation module is implemented in the python based controller. The results show that ABB provides similar response time with three servers to the no DDoS attack cases. Consequently, request completion rate increases suddenly with $\mathrm{ABB}$. A disadvantage is that it is very costly for per packet processing.

In [24], an approach to defend against DNS amplification attacks via traffic monitoring tool sFlow [25] was proposed by Aizuddin et al. The proposed system utilizes the features of SDN for defending the attack. In the proposed method, flow packets are collected and processed using sFlow. The header field values are checked to identify whether the flow is generated from the DNS server. The suspected flows are delivered to the controller for mitigation purpose. The topology of the scenario contains a controller, a switch, and two hosts. For simulation, an SDN based emulator Mininet [26] is used with Open vSwitch [27]. For traffic flow analysis, sFlow-RT is used. The results are compared with some existing approaches such as Rossow [28] and Huistra [29].

A "controller scheduling" algorithm named MutliSlot to defend against DDoS attacks was proposed in [30]. The proposed method depends on allocation strategy that is based on time slicing. This method consists of two modules: 1) DDoS detection module 2) MultiSlot algorithm module. The objective of the method is to segregate the flow requests from various switches. It utilizes different time slice allocation methods for each individual switch. Simulation is performed on Mininet and POX controller [31]. The effectiveness of MultiSlot is compared with two existing techniques i.e., MultiQ and SingleQ. The proposed scheduling method provides more protection to the internal switches that are affected by the attack indirectly. This advantage is more obvious as the the strength of the attack increases.

Chen et al. [32] proposed a flexible distributed architecture named FlexProtect to provide protection for multi-tenant data centres. FlexProtect utilizes the capabilities of SDN and NFV to defend against DDoS attacks. It works on network level. The detection and mitigation 
modules are implemented separately in the FlexProtect system. The detection module is placed near the service provider and mitigation module is placed near the edge routers. Both modules are deployed in the form of virtual network function (VNF). It reduces the detection overhead and the bandwidth consumption of the attack traffic. Further, authors also proposed an anti-spoofing protection mechanism called FP-SYN based on FlexProtect. According to the simulation results, FlexProtect can effectively alleviate the effect of attack and reduce extra length of the routing path. The result of FP-SYN also presents that it can identify the attackers with high accuracy.

Zheng et al. [33] designed a system to defend against DDoS attacks via adaptive correlation analysis. They called it "Reinforcing Anti-DDoS Actions in Realtime (RADAR)". The system is fabricated upon the "commercial off the shelf (COTS)" that is adopted as an SDN switch. It has no requirement of any alteration in the switch or any extra appliances. It is the first system deployed upon COTS switches that can detect different DDoS attacks. The RADAR system is implemented in Floodlight controller. The RADAR comprises of three components: collector, detector, and locator. The RADAR can identify different flooding attacks such as crossfire attacks, SYN flood, UDP flood, and DNS amplification attacks in real-time. The test is performed on both Mininet and hardware-based testbed. The performance is measured in the form of accuracy, delay, and overhead. The results present that the proposed system can discover the DDoS more efficiently with less delays and acceptable overhead.

In [34], an SDN based DDoS defense mechanism (FlowTrApp) for data centers was presented by Buragohain et al.. The proposed mechanism works on two parameters: "flow rate" and "flow duration of a flow". These parameters define that how much a legitimate user can send and how long. FlowTrApp tries to detect the DDoS attacks on web based applications. It relies on the rules set by administrator specific to application layer. One session per IP address is allowed for HTTP requests at a time. A Fattree topology is used to draw a data center scenario. An aggregation layer is taken in the FlowTrApp architecture in which all the switches are OpenFlow enabled. The performance of FlowTrApp is compared with an existing QoS based mechanism. Simulation results show that FlowTrApp permits less number of illegitimate packets to be passed through it in comparison to QoS mechanism. The proposed method is also compared with a load balancing attack mitigation method. Flow Trapp performs better than load balancing method. The results show that the Flow Trapp reduces the burden of the controller.

An SDN based defense model to defend against Slow HTTP DDoS attacks was proposed in [35]. The detection of slow DDoS attacks is troublesome because they act as low rate-benign traffic flows. The proposed defense model comprises a controller running a module called Slow HTTP DDoS Defense Application (SHDA), two OpenFlow devices, targeted web server. Further, the clients are classified into three groups of users i.e., malicious, slow, and normal. SHDA discovers whether a user is malicious or legitimate. SHDA establishes a threshold for HTTP requests to be completed. The requests that exceed the SHDA's threshold value are identified as malicious requests. For mitigation purpose, SHDA instructs the controller to make a fresh rule that blocks the malicious flow at forwarding device. The results show that the defense model blocks threats efficiently and allows the server to continue its normal operation.

Shtern et al. [36] proposed an architecture to mitigate the effect of application layer based slow and low DDoS. Their architecture utilizes SDN's adaptive capabilities for defense whenever required. A concept of 'shark tank' is also introduced in this scheme where probable malicious users are redirected. Shark tank is a module that analyses the attack activities and guides the system to know about the attack. Recently, in [37], authors presented an 
approach for detection and mitigation of slow HTTP attacks by utilizing SDN's flexible features. It provides flow based analysis of the network traffic and detects the attackers and isolates malicious traffic from the network.

In [38], Tripathi et al. analyzed the behavior of slow HTTP based DDoS attacks on most widely used web servers (Apache, Microsoft IIS, Nginx, and Lighttpd) and proposed a detection mechanism. Authors evaluated the performance of the detection system against two HTTP based attacks i.e., slow header and slow message body attacks. The proposed detection approach has two phases: training and testing based on probabilistic distribution of training and testing data. A distance named Hellinger between the training and testing probability distributions is used to detect the attacks.

Hirakawa et al. [39] proposed a defense method against HTTP based slow DDoS attacks (header attack, message body attack, and slow read attack). In this method the main focus is on the number of connections from an IP address and the duration time. Thus, if the number of connections from a specific IP address exceeds a threshold value, all the connections from that IP are disconnected. In [20] also, authors suggested a solution for detection of HTTP based slow DDoS attacks. Two parameters namely window size and delta time of the packet were used to analyze the traffic patterns generated by attack and mitigate it. In [40], Kemp et al. provided an approach to detect slow read attack by using different ML based classifiers using netflow based data.

Huang et al.[41] proposed a simple and efficient method named SDN One-packet DDoS Mitigation (SODM) to defend against DDoS attacks. This approach drops all one packet flows as soon as DDoS attack suspected. The network topology consists of OpenFlow switch, controller, and security analyzer. Security analyzer is used to analyze the traffic flow statistics and carry out some malicious indicators. After analyzed the traffic, attack indicators are sent to the controller to take proper actions. Among all the incoming flows, a single flow may be malicious that acts like a benign flow. SODM drops all one packet flows, if an attack is identified within a given observation period. This method provides efficient false positive rate and response rate. Accuracy of the proposed mechanism depends on the monitoring window size.

In [42], a dynamic policy based mechanism to mitigate the impacts of attacks on the customer networks was presented. Authors attempt to produce a "fine-grained and automated mitigation" system in Internet Service Provider (ISP) network by using SDN capabilities. It is based on the high level policies that ISPs have to enforce dynamically. The proposed system is based on user centric automated response that provides QoS service to the customers. The primary aim is to decrease the effect of attacks on the ISP customers. The global view of SDN helps to achieve this goal. It works on the requirements of customers accordingly. It supports multiple customers that are served by a single ISP. In the proposed approach, a mutual relationship between ISP and its customers is defined to handle the congestion induced by DDoS attacks. Throughput and jitter are utilized for performance measurement of the proposed approach. This mechanism allows policies to be adaptively updated based on customer's requirements. It provides quick response and attack mitigation.

Sahay et al. in 2017 [43] proposed a mitigation framework named autonomic DDoS mitigation framework (ArOMA) by using dynamic programmability and global view features of SDN. ArOMA provides a collaboration between ISP and its clients to provide an ondemand mitigation of DDoS threats. In this approach, the client side monitors the network traffic and detects the attacks while ISP side performs DDoS mitigation based on some policies. This method does not bring computational burden to ISP. The clients run their own attack detection module and generate alarms concerned alerts are reported towards ISP.

ACM Comput. Surv., Vol. 1, No. 1, Article 1. Publication date: January 2018. 
The proposed framework is validated using simulation and testbed experiments. ArOMA supports only a single client environment. The results conclude that ArOMA provides quick response for recovering benign traffic's performance. Classification of the traffic as benign and malicious is not reported in this work. ArOMA ensures that the video streaming service can maintain its efficiency while it is being attacked by flooding attacks. It also maintains Quality of Experience (QoE) and Quality of Service (QoS) metrics.

In [44], a collaborative mitigation mechanism for DDoS attacks using SDN was proposed. The authors developed a protocol called controller-to-controller (C-to-C) to provide a secure communication between controllers. The developed protocol permits the controllers to share the information with other controllers in different domains, and helps to notify them regarding a running attack. This activates an efficient notification for the running attacks on the path and filters the network traffic near the origin of the attack. This decreases the processing time and usage of network resources. Authors also created a C-to-C packet that is delivered to the controller by a detection engine. The packet contains three components such as data, certificate, and signature. Different functionality segments are installed on the upper side of controller. The testbed of the proposed mechanism is divided into three networks: source, intermediate and destination network. Simulation is performed on Mininet. The instances of Mininet emulating different networks are connected by GRE tunneling. In this approach, a node is simulated as detection engine. It can be placed in any of the network or above the network. The performance is evaluated in terms of dissemination delay and throughput. It shows the acceptable usage of CPU (35\%) and memory (25\%) and overhead.

Wang et al. in 2018 [45] proposed a mechanism named "Woodpecker" to detect and mitigate a new type of DDoS attack - link flooding using SDN capabilities. In the proposed mechanism a number of selected ordinary switches are upgraded to SDN enabled switches. With the help of global view provided by the controller Woodpecker locates the congested location and identifies whether the congestion is actually caused by link flooding. Woodpecker imposes traffic engineering as an application on the controller to mitigate the impacts of attack. The results show that the bandwidth utilization of congested links is reduced upto $50 \%$. The average packet loss rate and jitter are decreased.

\subsection{Machine learning based defense mechanisms}

In this section, ML based mechanisms [46] are analyzed that can detect the DDoS attacks. In recent years, ML has gained attention of many technologies as a promising technique. Various ML algorithms have been adopted for security purposes. Despite traditional networks, they are also being used to detect and mitigate the attacks in SDN. These algorithms are utilized as a classifier to classify the traffic into malicious and benign. Most commonly used algorithms are support vector machine (SVM) [47], neural network, naive bayes, k-means clustering, fuzzy logic, genetic algorithm, and self organizing map (SOM) [48] etc. These algorithms can be used to both detect and mitigate DDoS attacks. The analysis of ML based defense mechanisms is shown in Table 2. In [49], the authors have investigated some ML techniques to be used for DDoS defense in SDN. It suggests that all the algorithms have their own positives and negatives, so they can be used according to their requirements.

Quamar et al. [50] presented a "Deep learning based multi-vector DDoS detection" system in SDN network. This system is considered as a network application that is deployed on the controller. The proposed intrusion detection system incorporates stacked autoencoder (SAE) based deep learning approach to detect multi-vector DDoS attacks. Deep learning has been used for feature reduction. The detection system contains three modules i.e., Traffic Collector and Flow installer (TCFI), Feature Extractor (FE), and Traffic Classifier (TC). 
The proposed system relies on each packet for flow computation and attack detection instead of sampling flows, thus minimizing false positives. The features of the dataset produced from collected traffic traces were normalized using max-min normalization. Hping3 tool is used for launching different kinds of DDoS attacks on the testbed. The proposed system is compared with soft-max and neural network (NN) attack detection models. The SAE model shows better performance as compared to the soft-max and neural network model in terms of accuracy $(99.65 \%)$. However, SAE suffers from processing capabilities due to two reasons, first is feature extraction from every packet that can be handled by flow sampling and second is TCFI being developed on top of the controller.

In [51], Li et al. introduced a DDoS detection and defense model which is based on DL. Recurrent neural network (RNN), long short term memory (LSTM), and convolutional neural network $(\mathrm{CNN})$ are used in the detection model. The proposed model is applied to the OpenFlow switches. Performance of the defensive model is verified in a real-time environment by generating traffic through Spirent packet generator. The proposed model provides adaptability for making changes in DDoS detection approach in real-time.

Ahmed et al. [52] proposed a mitigation method to defend against DNS query based DDoS attacks using SDN features. The mitigation model is based on "Dirichlet Process Mixture Model (DPMM)" to differentiate the attack traffic from benign traffic. The proposed system is deployed on control plane. The SDN controller collects all the traffic from switches periodically. The model consists of three modules namely traffic statistics manager, learner component, and network resource manager. The first module captures the features of incoming flows from switches. Learner is responsible for detecting the malicious flows. The third module maintains a record of device's resource utilization. The switches are informed to update a new rule to block the traffic after detecting malicious flows. The proposed DPMM based model is compared with Mean-Shift algorithm based model to evaluate the efficiency. The results indicate that DPMM model performs with higher accuracy than Mean-Shift model.

An approach named FADM for defending against DDoS threats was proposed in SDN environment [53]. FADM achieves a decent efficiency and lightweight properties. FADM incorporates two modules i.e., detection and mitigation. In FADM, the network traffic statistics are analyzed by SDN controller using sFlow method. The proposed approach collects sufficient information for maintaining the desirable accuracy of the system. It cannot collect the information completely for high traffic rates. Current network features are extracted from the collected information. Proposed mitigation module depends on white-list and traffic migration. An entropy based method is utilized for evaluating the network features and SVM is used for identifying DDoS attacks. The response and performance of the attack detection can be potentially enhanced by combining the proposed approach with the other methods. Experimental evaluation outcomes conclude that FADM can provide accurate detection and effective mitigation of various DDoS threats. In addition, FADM is capable of recovering the network in a very short time.

A method (SDN-Anti-DDoS) [54] was proposed and demonstrated to detect DDoS attacks in a fast and efficient manner by Cui et al. The proposed method consists of four modules each serving a special purpose. An attack trigger detection module is implemented to give response against an attack quickly as well as decreases load on controllers and switches. Neural networks are used for the detection of the attacks. Furthermore, authors also proposed a traceback method utilizing capabilities of SDN to track down the attack route. A mechanism is designed and deployed on RYU controller to obstruct the attacks and perform flow table cleaning. The results show that SD-Anti-DDoS can quickly detect the malicious 
activity within one second and discover the source of the attack. The proposed detection trigger mechanism can respond more quickly against the attack than other existing periodic trigger methods. It also decreases burden of the CPU and the controller. Most importantly, SDN-Anti-DDoS supports different variants of OpenFlow protocol.

An approach was presented to detect and mitigate SMTP flood attacks in SDN [55]. A framework named FlowIDS is used as a detection module to identify anomalies in SMTP flows. Decision tree (DT) and deep learning (DL) algorithms are used to classify malicious and benign traffic accurately. It is combined with Suricata NIDS for controlling and monitoring the traffic flows. The testbed used for the evaluation of proposed technique consists of systems having 8 Core Xeon CPU with 16 GB RAM and 80 GB storage each. The simulations of the FlowIDS framework were conducted for DT and DL in a single site. As per the results, DL provides better bandwidth utilization and faster network recovery than DT. Simulation of FlowIDS with DL algorithm in multi-site may be considered as future work.

da Silva et al. [56] designed a framework titled ATLANTIC for defending against DDoS was designed by utilizing the SDN features. ATLANTIC combines the functionalities of detection, classification, and mitigation. This framework attempts to block the malicious flows from external networks. It consists of two phases: a lightweight processing phase and a heavyweight processing phase for monitoring and defending against attacks, respectively For first phase, entropy based analysis is used for fast detection. The classification is based on supervised and unsupervised ML algorithms. Anomaly detection framework comprises of a classification layer, statistical layer, and a network layer. The statistical layer collects traffic flow statistics and delivers it to the classification layer. An entropy based analysis is used for detecting variations in traffic features. SVM is used as a classifier and k-means is used for clustering. The experiment is performed considering two different attacks: port scanning and DDoS attack. ATLANTIC is deployed on the controller. SVM is shown to achieve accuracy of $88.7 \%$ with $82.3 \%$ precision. ATLANTIC minimizes the overhead of the proposed scheme on the controller. Ye et al. [57] also proposed a DDoS detection approach using SVM classifier. The proposed detection framework consists of flow status collection, features extraction, and classification of the extracted feature values. Authors implemented a feature extraction module to extract the features related to DDoS to train the classifier They have used flooding based attack traffic (TCP, UDP, and ICMP) to demonstrate the proposed detection approach. The results show that the approach gives an average accuracy of $95.24 \%$ and lower false alarm rate.

Lee et al. [58] examined the problem of integrating an "anomaly detection" development framework into current SDN deployments. They proposed a fully distributed application hosting architecture called Athena. The proposed framework facilitates good scalability as compared to previously developed SDN security frameworks. Athena is a software solution that provides an interface and some useful APIs for prototyping and generalizing different anomaly detection methods with minimal efforts required for programming. Authors have shown the effectiveness of proposed framework by considering three scenarios, a large-scale DDoS attack detector, Link Flooding Attacks (LFA) Mitigation, and Network Application Effectiveness (NAE). Only a DDoS scenario is considered in this work. In this scenario first, the DDoS detection model is created followed by feature validation phase then the testing is done at last. A clustering algorithm- k-means is used for DDoS detection. The main advantage of using Athena is that it requires fewer lines for coding a DDoS detection algorithm, scalable and incurs less processing overhead as compared to Spark and Hama.

Assis et al. [59] introduced Game Theory based on Holt-Winters and Digital Signature (GT-HWDS) against DoS/DDoS threats. The objective of the proposed system is to defend 
against the attacks on SDN controller. This protection system works on two methods i.e., HWDS system to identify the malicious activities, and GT-based solution facilitating the selection of an optimal defense strategy against an attack. The GT concept can be used in a more automatic way for mitigating the DDoS and other threats. In GT method, the issue is transformed into a game scenario of different players i.e., attackers and the protection mechanism. It has the capability of quick decision making for the DDoS attacks. GT-HWDS system contains three interactive modules such as Detection, Information, and Mitigation modules. The detection module analyses seven IP flow dimensions simultaneously for characterizing the traffic behavior. Primary benefit of the system is its operability in any SDN configuration. It does not require any specific configurations for mitigation purpose. The proposed mitigation module can be used as an autonomous approach with other detection modules. Fuzzy-GADS (Genetic Algorithm and Digital Signature) is combined with game theory method for performance evaluation. The Fuzzy-GADS is analyzed with six dimensional traffic flows. The genetic algorithm performs characterization of the traffic flows Fuzzy logic is used in detection of anomalies. The results show that GT-HWDS approach is more efficient and stronger than fuzzy logic based approach. It efficiently reduces saturation on SDN controller.

Chen et al. [60] designed a detection method based on SDN for DRDDoS (Distributed Reflection DoS) attacks based on amplification. A detection module is attached to SDN controller for detecting DRDDoS packets in the proposed architecture. This module consists of a traffic monitoring tool and a ML based classifier. An open source software tool i.e., Netmate is used to capture the network traffic. SVM is used to classify the traffic, it is trained to analyze DNS attack. In addition, it performs well with NTP attacks. It provides high accuracy. The detection module informs the controller about the malicious packets and instructs to block the attack. For experimental design, VMware ESXi is used to launch virtual machines with ONOS (Open Network Operating System) controller [61]. The method can detect and block both the attacks with less response time and blocking time. It is advantageous that controller reduces the burden of the detection module inspecting selected packets. This method can also detect unknown attacks.

Yan et al. [62] proposed a "DDoS Detection and Mitigation Framework (DDMF)" using features of SDN and Apache Spark. The objective of proposed framework is to discover and reduce the effects of DDoS in time. Apache Spark analyzes the network traffic more quickly. Capture Server, Detection Server (Cluster), and SDN Router Application, are three main components of DDMF. SDN Router Application is responsible for setting up the logic for flow rules of the packets. It controls the network and blocks the malicious flow entries. Capture Server captures the traffic flows and maintains a log file. A DDMF takes the advantage of SCP protocol to transfer the log file to Detection Server that makes sure the integrity of the transferred files. Detection module analyzes the log file and notifies the router application to block the malicious flows. For detection of the attacks, several methods such as neural network, entropy based, counting based can be used. The simulation of DDMF is performed on the testbed. DDMF blocks simulative DDoS flows automatically on the basis of analysis of the traffic.

He et al. [63] presented two filtered algorithms against DDoS threats to handle large network traffic. The algorithms utilize SDN capabilities to detect anomalies in the network pattern. The proposed algorithms are: "unsupervised cluster based feature selection" and "density peak based clustering with sampling adaption". The feature selection algorithm discards the redundant features in the dataset. It is suitable for continuous and discrete both features. Clustering is used for classifying the traffic into benign and anomalous data. 
The clustering algorithm provides better results in terms of runtime and memory efficiency. Evaluation shows better accuracy results.

Alshamrani et al. [64] proposed a defense system for defending a large variety of DDoS attacks in SDN. The defense system captures the traffic information from switches regularly and uses ML algorithm for classification. The DDoS detection module is based on the appropriate features selection of network traffic. Three algorithms such as ranker, genetic, and greedy algorithm are used for selecting a proper features subset. This system offers a good detection accuracy rate. Further, authors attempt to design other defense modules to mitigate two new attacks including misbehavior attack and newflow attack. The defense modules are executed over the controller. As per evaluated results, it reduces the attacker's capacity and maintains the services for normal users. It is more effective in terms of cost.

Liu et al. [65] presented a defense system named FL-GUARD (Floodlight Guard) to defend against DDoS in SDN. An anti-spoofing module of source IP is integrated with the Floodlight controller and the sFlow-RT collector component in the control layer. The attack detection and attack blocking modules are implemented in the application layer. The attack detection module uses C-SVM algorithm as a classifier to differentiate between normal and malicious flows. To block the attacks at the source port, the flow tables are assigned by utilizing the features of the SDN central control. According to simulation results, the proposed defense system provides a good accuracy by detecting the DDoS attacks effectively.

\subsection{Application specific/Collaborative defense mechanisms}

This section is available as online supplementary material.

\section{DEFENSE MECHANISMS FOR SDN AGAINST DDOS}

Despite SDN's centralized control of the entire network to detect and mitigate the DDoS attacks, it is still open to many types of DDoS attacks, which must be addressed. Due to the separation of control logic out of the forwarding devices, it makes SDN vulnerable to several security threats. These threats need to have a point of focus of the researchers and many commercial vendors to secure the networks. Many detection and mitigation mechanisms have been provided to mitigate the DDoS attacks for securing SDN. Some defense solutions proposed in the literature are discussed in this section. Table 3 presents various defense mechanisms of application-specific types. These solutions are classified into different categories such as data plane solutions, control plane solutions, switch-controller based collaborative solutions, collaborative intelligent switches, and integrated solutions on the basis of possible causes of the threats on the SDN planes.

\subsection{Defense against attack on data plane}

Data plane contains dumb switches that can help the attackers to be targeted. The OpenFlow switches in SDN are not capable to resolve the threat issues by their own. DoS/DDoS are the most convenient threats nowadays to damage the network. They can overload the SDN switches by flooding based attacks. The SDN switches also suffer from limited size of TCAM. These issues should be addressed to mitigate the DDoS attacks on the data plane. In this section, some existing detection and mitigation mechanisms are presented. The defense mechanisms should be quick enough to react against the attacks. The data plane defense requires additional appliances or modifications in the OpenFlow switches that may be costly. Thus, defense mechanisms should also be cost-effective. 


\begin{tabular}{|c|c|c|c|c|c|c|c|}
\hline Ref. & Defense mechanism & Intrusion type & $\begin{array}{l}\text { Targeted } \\
\text { resources }\end{array}$ & $\begin{array}{l}\text { Simulation } \\
\text { tools }\end{array}$ & Controller & Advantages & Limitations \\
\hline [30] & $\begin{array}{lr}\text { Multislot } & \text { (con- } \\
\text { troller scheduling } \\
\text { method } r \text { based } \\
\text { on time } & \text { slice } \\
\text { allocation) } & \\
\end{array}$ & $\begin{array}{l}\text { DDoS (No specific } \\
\text { type) }\end{array}$ & - & $\begin{array}{l}\text { POX, } \\
\text { Mininet }\end{array}$ & Single & $\begin{array}{l}\text { Provides better protection for } \\
\text { internal switches as compared } \\
\text { to SingleQ and MultiQ algo- } \\
\text { rithms. }\end{array}$ & $\begin{array}{l}\text { Not applicable for large } \\
\text { scale networks (queue main- } \\
\text { tenance overhead for each } \\
\text { switch makes the system } \\
\text { complex) }\end{array}$ \\
\hline [23] & $\begin{array}{l}\text { ABB (Adaptive } \\
\text { Bubble Burst) }\end{array}$ & $\begin{array}{l}\text { Service exhaustion } \\
\text { attacks (NTP, } \\
\text { HTTP, FTP) }\end{array}$ & $\begin{array}{l}\text { CPU/ } \\
\text { server }\end{array}$ & $\begin{array}{l}\text { POX } \\
\text { controller, } \\
\text { Real-time }\end{array}$ & Single & $\begin{array}{l}\text { Completely transparent to } \\
\text { client and server ends. Does } \\
\text { not require any modifications } \\
\text { at both ends, enhance the } \\
\text { availability of an specific } \\
\text { service during DDoS }\end{array}$ & $\begin{array}{l}\text { Very costly, high per packet } \\
\text { processing Overhead, not } \\
\text { a defense solution, more re- } \\
\text { sponse time }\end{array}$ \\
\hline [33] & $\begin{array}{l}\text { RADAR } \quad \text { (using } \\
\text { adaptive correla- } \\
\text { tion analysis) }\end{array}$ & $\begin{array}{lr}\text { Link } & \text { flood- } \\
\text { ing } & \text { (crossfire), } \\
\text { SYN flooding, } \\
\text { UDP/DNS amplifi- } \\
\text { cation attacks }\end{array}$ & $\begin{array}{l}\text { Bandwidth, } \\
\text { memory }\end{array}$ & $\begin{array}{l}\text { Flood- } \\
\text { light, } \\
\text { Mininet }\end{array}$ & Single & $\begin{array}{l}\text { Reduces response time of con- } \\
\text { troller, avoids single point of } \\
\text { failure and cascading failure, } \\
\text { does not require extra appli- } \\
\text { ances or any modifications }\end{array}$ & $\begin{array}{l}\text { In case of small network } \\
\text { topology with few switches } \\
\text { incurs more overhead }\end{array}$ \\
\hline$[24]$ & $\begin{array}{l}\text { Security-centric } \\
\text { SDN }\end{array}$ & $\begin{array}{l}\text { DNS amplification } \\
\text { attack }\end{array}$ & Memory & $\begin{array}{l}\text { Kinetic, } \\
\text { Mininet }\end{array}$ & Single & $\begin{array}{l}\text { Less response time, adaptive, } \\
\text { better throughput and less jit- } \\
\text { ter as compared to existing } \\
\text { methods }[28],[29]\end{array}$ & $\begin{array}{l}\text { It is not clear if this system } \\
\text { works for the NTP amplifi- } \\
\text { cation attacks }\end{array}$ \\
\hline [34] & $\begin{array}{l}\text { FlowTrApp (based } \\
\text { on flow statistics) }\end{array}$ & $\begin{array}{l}\text { Flooding attacks } \\
\text { (UDP, ICMP) }\end{array}$ & Bandwidth & $\begin{array}{l}\text { Flood- } \\
\text { light, } \\
\text { Mininet }\end{array}$ & Single & $\begin{array}{l}\text { Reduced burden of the Open- } \\
\text { Flow controller, reduced false } \\
\text { negative rate compared to a } \\
\text { QoS based mechanism, can de- } \\
\text { tect and mitigate variable rate } \\
\text { (low/high) DDoS }\end{array}$ & $\begin{array}{l}\text { Applicable only for flooding } \\
\text { attacks }\end{array}$ \\
\hline$[32]$ & $\begin{array}{l}\text { An anti-spoofing } \\
\text { protection mecha- } \\
\text { nism (FP-SYN) }\end{array}$ & SYN flooding & Memory & $\begin{array}{l}\text { Mininet2.2, } \\
\text { OVS2.3.1 }\end{array}$ & Single & $\begin{array}{l}\text { Reduces cost, reduces detection } \\
\text { overhead and bandwidth con- } \\
\text { sumption of the attack traffic }\end{array}$ & $\begin{array}{l}\text { Provides a defense for only } \\
\text { TCP-SYN attacks }\end{array}$ \\
\hline$[44]$ & $\begin{array}{l}\text { C-to-C protocol for } \\
\text { secure communica- } \\
\text { tion of controllers }\end{array}$ & ICMP flooding & Bandwidth & $\begin{array}{l}\text { POX, } \\
\text { Mininet }\end{array}$ & $\begin{array}{l}\text { Multi- } \\
\text { controller }\end{array}$ & $\begin{array}{l}\text { Reduced overhead, reasonable } \\
\text { resource utilization }\end{array}$ & $\begin{array}{l}\text { examined only for one } \\
\text { DDoS type }\end{array}$ \\
\hline
\end{tabular}




\begin{tabular}{|c|c|c|c|c|c|c|c|}
\hline Ref. & $\begin{array}{l}\text { Defense } \\
\text { mechanism }\end{array}$ & $\begin{array}{l}\text { Intrusion } \\
\text { type }\end{array}$ & $\begin{array}{l}\text { Targeted re- } \\
\text { sources }\end{array}$ & $\begin{array}{l}\text { Simulation } \\
\text { tools }\end{array}$ & Controller & Advantages & Limitations \\
\hline [43] & $\begin{array}{l}\text { ArOMA } \\
\text { (SDN-based } \\
\text { autonomic } \\
\text { mitigation } \\
\text { framework) }\end{array}$ & $\begin{array}{l}\text { Flooding } \\
\text { attacks } \\
\text { (UDP, } \\
\text { TCP-SYN, } \\
\text { ICMP) }\end{array}$ & $\begin{array}{l}\text { Bandwidth, } \\
\text { memory }\end{array}$ & $\begin{array}{l}\text { Ryu, } \\
\text { Mininet }\end{array}$ & $\begin{array}{l}\text { Multi- } \\
\text { controller }\end{array}$ & $\begin{array}{l}\text { Reduces workload } \\
\text { of controller and } \\
\text { switch }\end{array}$ & $\begin{array}{l}\text { QoS de- } \\
\text { graded, Sin- } \\
\text { gle customer } \\
\text { network } \\
\text { support }\end{array}$ \\
\hline [35] & $\begin{array}{l}\text { SDN- } \\
\text { Assisted } \\
\text { Slow HTTP }\end{array}$ & $\begin{array}{l}\text { Slow } \\
\text { HTTP } \\
\text { DDoS } \\
\text { attacks }\end{array}$ & $\mathrm{CPU}$ & NS3 & Single & $\begin{array}{l}\text { No computational } \\
\text { burden on ISP, } \\
\text { quick response, } \\
\text { reduces the damage } \\
\text { in the network }\end{array}$ & $\begin{array}{l}\text { May have } \\
\text { less detection } \\
\text { accuracy, } \\
\text { commu- } \\
\text { nication } \\
\text { overhead not } \\
\text { discussed }\end{array}$ \\
\hline [41] & $\begin{array}{l}\text { SDN One- } \\
\text { packet } \\
\text { DDoS Mit- } \\
\text { igation } \\
\text { (SODM) }\end{array}$ & $\begin{array}{l}\text { Flooding } \\
\text { attacks } \\
\text { (SYN, } \\
\text { ICMP) }\end{array}$ & $\begin{array}{l}\text { Bandwidth, } \\
\text { memory }\end{array}$ & Real time & Single & $\begin{array}{l}\text { Quick response to } \\
\text { attack }\end{array}$ & $\begin{array}{l}\text { Restricted } \\
\text { monitoring } \\
\text { window size }\end{array}$ \\
\hline$[42]$ & $\begin{array}{l}\text { Adaptive } \\
\text { policy } \\
\text { driven }\end{array}$ & $\begin{array}{l}\text { UDP, } \\
\text { TCP-SYN, } \\
\text { ICMP } \\
\text { flooding, } \\
\text { DNS am- } \\
\text { plification } \\
\text { attacks } \\
\end{array}$ & $\begin{array}{l}\text { Bandwidth, } \\
\text { memory }\end{array}$ & $\begin{array}{l}\text { Ryu, } \\
\text { Mininet }\end{array}$ & Single & $\begin{array}{l}\text { Quick response and } \\
\text { fast mitigation, re- } \\
\text { duces flow entries } \\
\text { in the switches, dy- } \\
\text { namically instanti- } \\
\text { ated and adaptive } \\
\text { security policies }\end{array}$ & $\begin{array}{l}\text { Detection } \\
\text { mechanism } \\
\text { not discussed }\end{array}$ \\
\hline [45] & $\begin{array}{l}\text { Woodpecker } \\
\text { (Policy } \\
\text { based ap- } \\
\text { proach) }\end{array}$ & $\begin{array}{l}\text { Link flood- } \\
\text { ing (UDP } \\
\text { flood) }\end{array}$ & Bandwidth & $\begin{array}{l}\text { POX, } \\
\text { Mininet }\end{array}$ & Single & $\begin{array}{l}\text { Reduces bandwidth } \\
\text { utilization of con- } \\
\text { gested link }\end{array}$ & $\begin{array}{l}\text { Applicable } \\
\text { to a spe- } \\
\text { cific type of } \\
\text { attack }\end{array}$ \\
\hline
\end{tabular}

In [66], Xu et al. proposed a model to defend against SDN based table-overflow attacks. For solving the issue, a mathematical mechanism is designed according to SDN topology. Probable victim switches are defined based on mathematical formulation. A switch with very less vacancies is selected as a target switch (hot switch) for the attack. Attackers use a switch placed at midway in place of endpoints. A monitoring mechanism with three traffic flow features are defined to discover the attack and reveal the attackers. After monitoring the attack traffic, a token bucket algorithm based defense model is implemented in the controller. This defense model ensures stable transmission rate of normal clients and limits the rate of attackers. Both monitoring and defense models are implemented into the actual routing applications of SDN. The effectiveness of monitoring mechanism is evaluated WAN, LAN, and data center frameworks. For simulation of mitigation model, Open vSwitch as SDN switches and OpenDaylight controller [67] are used. The results show that proposed method performs effectively by reducing the attack rate. 


\begin{tabular}{|c|c|c|c|c|c|c|c|}
\hline Ref. & Defense mechanism & Intrusion type & $\begin{array}{l}\text { Targeted re- } \\
\text { sources }\end{array}$ & $\begin{array}{l}\text { Simulation } \\
\text { tools }\end{array}$ & Controller & Advantages & Limitations \\
\hline$[62]$ & $\begin{array}{l}\text { DDMF (Big data } \\
\text { technologies such } \\
\text { as Apache Spark } \\
\text { based traffic analy- } \\
\text { sis) }\end{array}$ & ICMP flooding & Bandwidth & Real-time & Single & Fast traffic processing & $\begin{array}{l}\text { Simulated for one type of } \\
\text { DDoS traffic, flow based de- } \\
\text { tection that may compro- } \\
\text { mise with accuracy }\end{array}$ \\
\hline$[53]$ & $\begin{array}{l}\text { FADM (entropy } \\
\text { based analysis for } \\
\text { feature selection } \\
\text { and SVM for } \\
\text { classification) }\end{array}$ & $\begin{array}{l}\text { Flooding attacks } \\
(\text { SYN, UDP, ICMP) }\end{array}$ & $\begin{array}{l}\text { Bandwidth, } \\
\text { memory }\end{array}$ & $\begin{array}{l}\text { POX, } \\
\text { Mininet }\end{array}$ & Single & $\begin{array}{l}\text { Less response time, high accu- } \\
\text { racy, quick recovery }\end{array}$ & $\begin{array}{l}\text { More recovery delay in case } \\
\text { of SYN flood than other } \\
\text { flooding attacks }\end{array}$ \\
\hline [59] & $\begin{array}{l}\text { Game theory } \\
\text { (GT)-Holt-Winters } \\
\text { for Digital Signa- } \\
\text { ture (HWDS) }\end{array}$ & $\begin{array}{l}\text { UDP flooding, } \\
\text { Portscan }\end{array}$ & Bandwidth & Real-time & Single & $\begin{array}{l}\text { Operable with any SDN config- } \\
\text { uration for mitigation }\end{array}$ & $\begin{array}{l}\text { Performed with only UDP } \\
\text { based DoS/DDoS }\end{array}$ \\
\hline$[52]$ & $\begin{array}{lr}\text { Dirichlet } & \text { process } \\
\text { mixture } & \text { model } \\
(\mathrm{DPMM}) & \text { based } \\
\text { clustering } & \end{array}$ & $\begin{array}{l}\text { DNS query-based } \\
\text { DDoS (DNS ampli- } \\
\text { fication, DNS flood- } \\
\text { ing) }\end{array}$ & $\mathrm{CPU}$ & - & Single & $\begin{array}{l}\text { Less delays, acceptable over- } \\
\text { head, increases accuracy, does } \\
\text { not need modification or extra } \\
\text { appliances }\end{array}$ & No mitigation provided \\
\hline$[64]$ & $\begin{array}{l}\text { Machine learning } \\
\text { classification algo- } \\
\text { rithm(sequential } \\
\text { minimal optimiza- } \\
\text { tion) }\end{array}$ & $\begin{array}{l}\text { Flooding attacks } \\
\text { (UDP, TCP-SYN, } \\
\text { ICMP), misbehav- } \\
\text { ior attack, newflow } \\
\text { attack }\end{array}$ & $\begin{array}{l}\text { Bandwidth, } \\
\text { memory }\end{array}$ & $\begin{array}{l}\text { POX, } \\
\text { Mininet }\end{array}$ & Single & $\begin{array}{l}\text { Supports wired or wireless both } \\
\text { infrastructure, useful for cost- } \\
\text { benefit analysis for mitigation }\end{array}$ & $\begin{array}{l}\text { Processing overhead not } \\
\text { discussed }\end{array}$ \\
\hline$[54]$ & $\begin{array}{l}\text { SD-Anti-DDoS } \\
\text { (neural network } \\
\text { based detection) }\end{array}$ & $\begin{array}{l}\text { Flooding attacks } \\
\text { (UDP, TCP-SYN, } \\
\text { ICMP) }\end{array}$ & $\begin{array}{l}\text { Bandwidth, } \\
\text { memory }\end{array}$ & $\begin{array}{l}\text { Ryu } \\
\text { Mininet }\end{array}$ & Single & $\begin{array}{l}\text { Fast response of detection, re- } \\
\text { duces controller load, no need } \\
\text { to add extra hardware. }\end{array}$ & $\begin{array}{l}\text { TFN2k tool is used to pro- } \\
\text { duce the traffic that is out- } \\
\text { dated nowadays. }\end{array}$ \\
\hline$[51]$ & $\begin{array}{l}\text { Deep learning } \\
\text { based }\end{array}$ & $\begin{array}{l}\text { Flooding attacks } \\
\text { (UDP, } \\
\text { ARP, SYN, } \\
\text { PingofDeath) }\end{array}$ & $\begin{array}{l}\text { Bandwidth, } \\
\text { memory }\end{array}$ & - & Single & $\begin{array}{l}\text { Reduces dependence on the } \\
\text { hardware and software, easy to } \\
\text { adapt the changes in real-time }\end{array}$ & Processing overhead \\
\hline
\end{tabular}




\begin{tabular}{|c|c|c|c|c|c|c|c|}
\hline Ref. & Defense mechanism & Intrusion type & $\begin{array}{l}\text { Targeted re- } \\
\text { sources }\end{array}$ & $\begin{array}{l}\text { Simulation } \\
\text { tools }\end{array}$ & Controller & Advantages & Limitations \\
\hline [55] & $\begin{array}{l}\text { FlowIDS (Deep } \\
\text { learning based) }\end{array}$ & $\begin{array}{l}\text { SMTP flooding at- } \\
\text { tack }\end{array}$ & Bandwidth & $\begin{array}{l}\text { ONOS, } \\
\text { Mininet }\end{array}$ & Single & $\begin{array}{l}\text { Quick detection and mitiga- } \\
\text { tion (by reducing network band- } \\
\text { width consumption }\end{array}$ & $\begin{array}{l}\text { Applicable for a specific } \\
\text { type of DDoS, unable to } \\
\text { simulate on multi-site }\end{array}$ \\
\hline [50] & $\begin{array}{ll}\text { Deep } & \text { learning } \\
\text { based } & \end{array}$ & $\begin{array}{l}\text { Flooding } \text { attacks } \\
\text { (ICMP, } \\
\text { TCP) }\end{array}$ & $\begin{array}{l}\text { Bandwidth, } \\
\text { memory }\end{array}$ & $\begin{array}{l}\text { POX, Real- } \\
\text { time }\end{array}$ & Single & $\begin{array}{l}\text { High accuracy with low false } \\
\text { positives }\end{array}$ & $\begin{array}{l}\text { Per packet processing over- } \\
\text { head for feature extraction, } \\
\text { increases burden of the con- } \\
\text { troller }\end{array}$ \\
\hline [65] & FL-GUARD & ICMP flooding & Bandwidth & $\begin{array}{l}\text { Floodlight, } \\
\text { Mininet }\end{array}$ & Single & $\begin{array}{l}\text { High detection accuracy, in- } \\
\text { creases flexibility, other busi- } \\
\text { ness of the abnormal hosts will } \\
\text { not be affected. }\end{array}$ & $\begin{array}{l}\text { Evaluation of all the per- } \\
\text { formance metrics are not } \\
\text { shown. }\end{array}$ \\
\hline [63] & $\begin{array}{lr}\begin{array}{l}\text { Clustering } \\
\text { anomaly }\end{array} & \text { detec- } \\
\text { tion (density } & \text { peak } \\
\text { clustering } & \text { with } \\
\text { unsupervised } & \text { clus- } \\
\text { ter based feature } \\
\text { selection) }\end{array}$ & $\begin{array}{l}\text { Information gath- } \\
\text { ering attack, DoS, } \\
\text { user to root, re- } \\
\text { mote to local at- } \\
\text { tacks (contained in } \\
\text { KDDCup99) }\end{array}$ & $\begin{array}{l}\text { Server, } \\
\text { computer's } \\
\text { resources } \\
\text { (memory, } \\
\text { processing } \\
\text { power) }\end{array}$ & $\begin{array}{l}\text { MINE, } \\
\text { Scikit-learn, } \\
\text { Numpy, } \\
\text { SciPy, Mat- } \\
\text { plotlib }\end{array}$ & Single & $\begin{array}{l}\text { Quick mitigation, reduces re- } \\
\text { dundant features, can handle } \\
\text { high dimensional and unlabeled } \\
\text { network data }\end{array}$ & $\begin{array}{l}\text { Clustering algorithms may } \\
\text { not give the accurate detec- } \\
\text { tion results. }\end{array}$ \\
\hline [58] & $\begin{array}{l}\text { Athena/DDoS } \\
\text { anomaly detec- } \\
\text { tion algorithm } \\
\text { (k-means, logistic } \\
\text { regression) } \\
\end{array}$ & $\begin{array}{l}\text { DDoS flooding at- } \\
\text { tacks }\end{array}$ & Bandwidth & $\begin{array}{l}\text { ONOS, } \\
\text { Mininet }\end{array}$ & $\begin{array}{l}\text { Multi- } \\
\text { controller }\end{array}$ & $\begin{array}{l}\text { Highly scalable, reduces com- } \\
\text { putational time for detection, } \\
\text { reduces programming effort }\end{array}$ & $\begin{array}{l}\text { Flow handling overhead, } \\
\text { classification accuracy may } \\
\text { be imperfect }\end{array}$ \\
\hline$[56]$ & ATLANTIC & $\begin{array}{l}\text { TCP-SYN attack, } \\
\text { Port Scan }\end{array}$ & Memory & $\begin{array}{l}\text { Floodlight, } \\
\text { Mininet }\end{array}$ & Single & $\begin{array}{l}\text { Reduces the overhead of the } \\
\text { overall detection mechanism, } \\
\text { provides human intervention in } \\
\text { case of automated modules not } \\
\text { working }\end{array}$ & $\begin{array}{l}\text { k-means can affect the de- } \\
\text { tection accuracy. }\end{array}$ \\
\hline [60] & $\begin{array}{l}\text { SDN and machine } \\
\text { learning technique } \\
(\mathrm{SVM}) \text { based detec- } \\
\text { tion }\end{array}$ & $\begin{array}{l}\text { Amplification } \\
\text { attacks (DNS, } \\
\text { NTP) }\end{array}$ & $\begin{array}{l}\text { Memory, } \\
\text { CPU }\end{array}$ & $\begin{array}{l}\text { ONOS, Real- } \\
\text { time }\end{array}$ & Single & $\begin{array}{l}\text { Can detect known or unknown } \\
\text { attacks, reduces the burden of } \\
\text { detection module, high scalabil- } \\
\text { ity }\end{array}$ & $\begin{array}{l}\text { False positive/false nega- } \\
\text { tive measures and process- } \\
\text { ing overhead not discussed. }\end{array}$ \\
\hline
\end{tabular}


Durner et al. [68] introduced DoS attacks against data plane and their effects in SDN. Authors proposed a statistical approach to detect attacks and a lightweight mitigation method to stop the malicious flows. It focuses on table overflow attacks in switches due to flooding attacks. The detection approach is based on analysis of header fields in the flow tables. It maintains a table of headers of suspected attackers using hashing. Based on that table, new rules are defined to handle or block the attack. Simulations and testbed experiments have been used to analyze the performance of proposed approach. The detection method is performed using OMNeT ++ tool on control plane level. The results show that attacks are detected efficiently and reliably with less false positives. The performance can be increased with a proper feature selection. Main limitation of this approach is that it is unable to identify the attackers who alter all header fields simultaneously.

\subsection{Defense against attack on control plane}

The control plane provides complete visibility of the SDN network. The complete functionality of the network may be disturbed, in the case of control plane breaks down. Because of having a centralized policy, an SDN controller is the most convenient to be targeted by the DDoS threats. Therefore, it is necessary to protect the controller by utilizing some appropriate detection and mitigation mechanisms. All the proposed defense mechanisms try to minimize the controller resource saturation produced by the DoS/DDoS attacks. In this section, some defense mechanisms have been discussed. The detection mechanisms should use quick response methods for identifying the attacks.

Mousavi et al. [69] proposed a system to detect DDoS attacks against controllers in its early stages. The proposed system uses controller's functionalities to protect the SDN network. It aims to quickly detect the attack and in order to provide a proper mitigation solution before the controller goes off. An entropy method based on destination address is used for detection of the attacks. Entropy is calculated using two factors i.e., defined window size and a threshold value in the proposed method. A functionality of collecting the destination IP addresses is integrated in the controller. An intrusion is reported if a certain threshold of computed entropy is crossed and vice versa. This method provides lightweight and fast detection of the malicious activities. The number of hosts can be dynamically changed for the proposed solution. The performance is tested on UDP and TCP traffic. However, the proposed solution also supports ICMP traffic. Major benefit of this solution is adaptability. The parameters used in the proposed algorithm can be modified according to targeted results in real-time. This is the first solution that is based on entropy for detecting the DDoS attacks in the SDN controller. A limitation is that the attacks cannot be identified against the whole network. It was designed only for a single controller architecture.

In [70], another entropy based approach was proposed by Sahoo et al. to detect low rate attacks at the controller. Authors used generalized entropy and information distance between different probability distributions as detection metrics. With the help of extracted statistical features from switch flow tables an alarm is indicated that shows probable DDoS attack in early stages. The proposed approach gives fast and accurate detection rate when compared to Mousavi's approach [69].

Wang et al. [71] proposed a lightweight and quick DoS defense mechanism called "SDNManager". The proposed system consists of five modules i.e., monitor, forecast engine, checker, updater, and storage service. The system analyzes the flow statistics, forecasts flow bandwidth changes based on these statistics, and updates the network accordingly. SDNManager applies a dynamic-time-series (DTS) model to improve bandwidth prediction accuracy. SDNManager is implemented on the control plane. It is valid for all types of DoS attacks.

ACM Comput. Surv., Vol. 1, No. 1, Article 1. Publication date: January 2018. 
Authors also proposed a dynamic controller scheduling (DCS) strategy in a multi-controller environment. The DCS strategy confirms the global network state optimization and defense efficiency. It assigns the controllers to switches dynamically according to the controller load. The average response time of the controller can be reduced by balancing an optimal mapping between controllers and switches. The defense scheme attempts to avoid both single point and cascading failure of controllers. For implementation purpose, a topology is used that consists of eight physical servers and four pica8 switches. The results show that forecast engine performs better than another forecasting model (ARCH). The effects of SDNManager is compared with the defense systems named SGuard and FloodGuard. SDNManager performs better in terms of bandwidth usage and CPU utilization. The DCS efficiency is evaluated using a data center topology containing 720 switches and 3456 host users with 30 Floodlight controllers. It is observed by the results that response time using DCS is very less than without DCS strategy. It includes some overhead that is in a minor range.

In [72], an approach (PATMOS) to mitigate DDoS attacks in multi-controller SDN environment using clustering of controllers was proposed. PATMOS involves three primary functions i.e., searching for bottlenecks, leader (controller) election, and composition. First, overwhelmed controllers are searched. Second, a controller is selected as the leader to coordinate among the controllers. Lastly, controllers are clustered for mitigating DDoS collaboratively. A genetic algorithm is utilized that finds the highest count of controllers in each cluster for handling the DDoS traffic. It helps to optimize the resource being used, thereby increasing network uptime. Five scenarios are considered for experiment and validation of the proposed approach. The first scenario evaluates the controller's normal behavior. In other scenarios, different number of controllers are used to identify the clusters with PATMOS. The effectiveness of the approach is computed based on metrics like CPU usage, latency, throughput, and total received packets.

Zhang et al. [73] proposed a dynamic queue based method i.e., multi-layer fair queuing (MLFQ) to mitigate controller's resource saturation attack. This queue management system encourages to fairly share the controller's resources. These dynamic queues can be expanded in case for attack traffic and can be aggregated for benign message requests.

\subsection{Defense against control plane bandwidth saturation}

The control plane bandwidth is the most concerned target of the DDoS attacker after SDN controller.

In [74], a defense model named SLICOTS to mitigate TCP-SYN flooding attacks by utilizing the SDN capabilities was presented. The mentioned DDoS attack reduces controller's performance. SLICOTS is employed in the control plane. It observes the running flows of requests and prevents the malicious requests in an effective manner. It is efficient for functioning only when controller behaves in a reactive manner. It informs the switches to block the malicious packets after identified an abnormal request. The effectiveness of SLICOTS is compared with a security model OPERETTA and ordinary SDN. It provides better results than OPERETTA based on metrics like response time, detection time, and CPU utilization. SLICOTS does not allow dropping of benign requests.

A framework named "AVANT-GAURD" that provides more defensive power to control plane was proposed in [75]. This framework expands the forwarding plane for completing the TCP handshake process with the TCP source and communicates. In addition to this forwarding plane is only allowed to communicate with the controller. Thus, if the handshake process is successfully completed, forwarding plane permits TCP connection establishment 
after informing the destination. A significant and unavoidable delay is introduced because of TCP connections. This framework requires reprogramming of SDN switches in order to add custom features to the forwarding plane.

\subsection{Defense by collaborative intelligence in switches}

In SDN, the controller is responsible for controlling all the switches and taking routing decisions. Thus, if switches do not find any matching flow entries for incoming packets in its flow table, all the packets are forwarded to controller. The switches are just simple forwarding devices. These switches are called as dumb switches as they cannot take decisions on their own. This characteristic introduces a large communication overhead and delay until the attack detection. This makes the controller overloaded and control channel congested. To overcome this issue, some researchers have proposed collaborative intelligence between switches and controller. Therefore, the switches also can take appropriate actions in detecting the malicious activities. This intelligence in simple forwarding switches may reduce the burden of the controller and control plane bandwidth. Some of these defense solutions are discussed in this section.

Han et al. [76] proposed a cross-plane DDoS attack defense framework named OverWatch. It accomplishes collaborative intelligence between forwarding devices and controller. This proposed framework includes two key methods i.e., attack detection and reaction. The detection system consists of a coarse-grained sensor and an actuator for flow monitoring on the data plane and a fine-grained ML based classifier on the control plane. The defense functionalities are split across forwarding and control planes to discover and mitigate the DDoS attacks on different levels. The performance of proposed framework is evaluated using a modified FPGA-based (Altera EP4SGX180) OpenFlow switch and a modified Ryu controller. Testbed of the experiment is performed using up to eight laptop hosts representing DDoS attackers, victims, and normal traffic generators, respectively. Experimental results show the efficiency of the defense system with high detection accuracy and real-time DDoS attack reaction. This method reduces communication overhead on SDN southbound interface.

Kalkan et al. [77] proposed a statistical and packet based approach called SDNScore to provide defense against DDoS attacks in SDN infrastructure. In the proposed approach, switches are embedded with some intellectual features with the packet forwarding rules to take a decisive action. SDNScore is a hybrid mechanism that works on collaboration between switches and controller. The architecture of SDNScore comprises modules i.e. profile, actuator, comparator, scorer, and pair-profiler. First four modules are situated on the switch and pair-profiler is implemented on the controller. All the modules coordinate with each other for detecting DDoS attacks. The proposed approach is motivated by a statistical filtering approach (PacketScore). A score value is calculated using attributes of packets and compared with a threshold. Based on this score, packets are dropped or forwarded. The proposed method can also identify new unknown DDoS attacks. It refines the malicious packets with the help of packet based analysis instead of blocking all the packets of a flow. The simulation outcomes prove the effectiveness of the proposed approach over entropy based DDoS detection method.

A new stateful approach (StateSec) to protect communication endpoints from DDoS attacks in SDN environment was presented by Boite et al. [78]. The idea behind StateSec is to develop stateful data plane API for SDN with the help of OpenState specification [79]. It attempts to unload the controller and control channel by assigning local decision capabilities to the switches. In this approach, the switches work more smartly than classical SDN switches. Finite state machines are implemented inside the switches to achieve this

ACM Comput. Surv., Vol. 1, No. 1, Article 1. Publication date: January 2018. 
goal. The complete defense idea depends on monitoring, detection, and mitigation of the attack traffic. Monitoring and detection functions are implemented inside the switches and mitigation is handled by the controller. A tool sFlow is integrated with Open vSwitch to analyze the incoming traffic. An entropy-based algorithm is used for detecting anomalies. The proposed method can detect multiple types of DDoS attack traffic. StateSec gives more efficient results in terms of detection rate and overhead on the control plane.

\subsection{Integrated mechanisms for DDoS mitigation}

Most of the proposed DDoS mitigation approaches address a particular SDN DDoS threat issue i.e., switch overflow, controller saturation or controller bandwidth congestion. In this section, some mitigation solutions are shown that are able to reduce the impact of the mentioned threats simultaneously. By avoiding all the issues, it can increase the SDN network's performance efficiently.

Dridi et al. [80] proposed an approach named SDN-Guard to protect the SDN network against DoS attacks. It attempts to reduce the effects of DoS attacks on SDN controller, controller-switch bandwidth, and switch memory usage. This approach depends upon an Intrusion Detection System (IDS). SDN-Guard is placed over the controller as a security application. IDS sends a notification to this security module when it finds a malicious behavior. Based on this alert, SDN-Guard module takes suitable decisions to mitigate the attacks. Because IDS handles all the traffic flows of network, it may get overloaded. Therefore, primary aim of this approach is to find an optimal place for IDS, and to reduce the packet flows sent to IDS by switches. The results show that SDN-Guard minimizes the effect of DoS, and minimizes the controller, switch memory overloading and switch-controller channel consumption up to $32 \%$. SDN-Guard uses traffic sampling to minimize packet loss and RTT while the network is going through DoS attack.

SDN and cloud are being used together to provide new ideas to design the network in programmable and portable manner. Security of SDN based cloud is also a challenge that must be solved. To resolve this issue, Chen et al. utilized a ML based classifier called extreme gradient boosting (XGBoost) to detect the DDoS attack in [81]. XGBoost is implemented in the SDN controller as a detection module. Authors focused on the control channel congestion and controller resources saturation threats. In the SDN based cloud scenario, different clouds are connected with each other. One cloud containing switches behaves as malicious cloud and targets to SDN devices in another cloud. Results show that XGBoost gives better accuracy and lower false positive rate as compared to other classifiers (Random Forest, SVM and Gradient based decision tree).

A flow migration defense (FMD) approach to protect the SDN network was suggested in [82]. Main idea is based on the migration of flooding requests from a master controller to a slave controller. FMD is implemented in the controller and does not require any change in network. In this mitigation method, controller and switch-controller channel are protected from DoS threats. In a normal scenario, the traffic requests are normally handled by the master controller. Upon detecting an attack, the suspected flows are migrated to the slave controller for processing. This migration of flows between controllers can protect the switch-to-master controller channel. The migrated requests are transferred to the master controller for further processing at a limited rate. It handles these requests with a dynamic adjustment of the requests depending on its actual workload. The performance of the FMD is evaluated in Mininet using Ryu controller. The authors also proposed an "adaptive rate adjustment (ARA)" method to gain a dynamic adjustment to handle flooding requests having no risk of overloading. The results are compared with existing approaches such 
as MLFQ and FloodDefender. FMD performs better in terms of response time, interface congestion, mitigation time, and packet loss ratio.

Wang et al. in 2015 [83] proposed a defense mechanism "Flood-Guard" against DoS attacks to avoid overloaded switch, control channel bandwidth, and the congested controller. Authors proposed a module named proactive flow rule analyzer that acts as a controller. This module controls all the new incoming packets in place of the controller during the attack. It works on the idea of dynamically changing of flow rules at runtime. It attempts to reduce the traffic burden on the overloaded controller. The proactive flow rule analyzer is not always efficient in providing accurate derivations. This approach was tested using both simulation and testbed. However, proposed approach may result in enhanced delay in processing the data packets that increases the time of setting up the new rules.

Furthermore, the security mechanisms are analyzed on the basis of threat cause and its impacts on the different planes. This analysis is shown in Table 4.

\section{PERFORMANCE EVALUATION}

Proposed different DDoS defense frameworks and solutions can be compared by evaluating some standard performance metrics. These performance metrics are:

(1) Classification metrics: Performance of attack/non attack traffic classification approach is measured by some parameters i.e., recall, precision, F-measure, accuracy, and ROC curve. These parameters are computed with the help of outcomes that are true positive, false positive, false negative, and false positive rates.

(2) Other performance metrics: The complete network can be analyzed by evaluating some important parameters. These parameters are end to end delay, CPU and memory usage, throughput, communication overhead, packet loss ratio, and network response time.

\section{RESEARCH CHALLENGES AND ISSUES}

We have identified several research issues from our extensive study. Most importantly, both detection and mitigation modules are essential requirements for securing a network. There are various research challenges and issues that need to be discussed and addressed for complete adoption of SDN technology.

\subsection{Security for SDN switches}

SDN switches have very limited memory (TCAM) to store the flow rules for the new incoming traffic. Due to storage constraints, switches gain attention of DDoS flooding attackers. Flooding attacks send a large number of packets aimed to consume all the storage of the flow tables in switches. Exhaustion of SDN switches can also interrupt the functionality of network. Just because controller is the main component of SDN, most of the work has been done for providing security to the controller. However, security of the switches used in the data plane must be studied in the similar manner.

\subsection{Cost for additional hardwares}

Researchers have reported few security suggestions and countermeasures for data plane security. These data plane defense proposals require modifications in the OpenFlow switches or usage of additional specific appliances. This increases the cost of setting up the SDN network. Therefore, some mechanisms should be proposed to overcome issues related with data plane security, while minimizing the overall network setup cost. 
Table 3. Defense mechanisms against DDoS for SDN

\begin{tabular}{|c|c|c|c|c|c|c|c|}
\hline Ref. & Defense mechanism & Intrusion type & $\begin{array}{l}\text { Targeted re- } \\
\text { sources }\end{array}$ & $\begin{array}{l}\text { Simulation } \\
\text { tools }\end{array}$ & Controller & Advantages & Limitations \\
\hline$[66]$ & $\begin{array}{l}\text { Using token bucket } \\
\text { algorithm }\end{array}$ & $\begin{array}{l}\text { Table overflow at- } \\
\text { tack }\end{array}$ & Memory & $\begin{array}{l}\text { OpenDay- } \\
\text { light, } \\
\text { Real-time }\end{array}$ & Single & Efficient in accuracy & - \\
\hline [68] & $\begin{array}{l}\text { Statistical based de- } \\
\text { tection }\end{array}$ & $\begin{array}{l}\text { Table-overflow at- } \\
\text { tack }\end{array}$ & Memory & $\mathrm{OMNeT}++$ & - & $\begin{array}{l}\text { More comprehensive approach } \\
\text { as it covers all the header fields, } \\
\text { efficient detection }\end{array}$ & $\begin{array}{l}\text { Cannot detect the attacks } \\
\text { that involve altering all the } \\
\text { headers simultaneously }\end{array}$ \\
\hline [69] & $\begin{array}{l}\text { Entropy based } \\
\text { DDoS detection }\end{array}$ & $\begin{array}{l}\text { Flooding attacks } \\
\text { (UDP, TCP, SYN) }\end{array}$ & $\begin{array}{l}\text { Bandwidth, } \\
\text { memory }\end{array}$ & $\begin{array}{l}\text { Pox, } \\
\text { Mininet }\end{array}$ & Single & $\begin{array}{l}\text { Reduces communication over- } \\
\text { head on southbound interface }\end{array}$ & $\begin{array}{l}\text { No prevention countermea- } \\
\text { sure considered, supported } \\
\text { only for a single con- } \\
\text { troller architecture addi- } \\
\text { tional overhead on the con- } \\
\text { troller }\end{array}$ \\
\hline$[71]$ & $\begin{array}{l}\text { SDNManager } \\
\text { (based on band- } \\
\text { width prediction) }\end{array}$ & $\begin{array}{l}\text { DoS attacks (valid } \\
\text { for all types) }\end{array}$ & $\begin{array}{l}\text { Bandwidth, } \\
\text { memory, } \\
\text { CPU }\end{array}$ & $\begin{array}{l}\text { Floodlight, } \\
\text { Real-time }\end{array}$ & $\begin{array}{l}\text { Multi- } \\
\text { controller } \\
\text { environ- } \\
\text { ment }\end{array}$ & $\begin{array}{l}\text { Reduces workload of controller } \\
\text { and control channel, smart } \\
\text { switches, reduces overhead of } \\
\text { controller, high detection rate }\end{array}$ & $\begin{array}{l}\text { Not realized the attacks } \\
\text { from multiple attacking ma- } \\
\text { chines (only DoS) }\end{array}$ \\
\hline$[72]$ & PATMOS & $\begin{array}{l}\text { DDoS (No specific } \\
\text { type mentioned) }\end{array}$ & - & $\begin{array}{l}\text { POX, } \\
\text { Mininet }\end{array}$ & $\begin{array}{l}\text { Multi- } \\
\text { controller }\end{array}$ & $\begin{array}{l}\text { Provides better results using } \\
\text { more controllers in the cluster }\end{array}$ & $\begin{array}{l}\text { Can lead to security (inter- } \\
\text { ception) problems between } \\
\text { controllers in cluster }\end{array}$ \\
\hline [82] & $\begin{array}{l}\text { FMD (based on } \\
\text { flow migration) }\end{array}$ & $\begin{array}{l}\text { DoS (No specific } \\
\text { type mentioned) }\end{array}$ & - & $\begin{array}{l}\text { Ryu, } \\
\text { Mininet }\end{array}$ & $\begin{array}{l}\text { Multi- } \\
\text { controller }\end{array}$ & $\begin{array}{l}\text { Reduces control plane \& con- } \\
\text { troller congestion }\end{array}$ & $\begin{array}{l}\text { May cause problems be- } \\
\text { tween controllers }\end{array}$ \\
\hline$[74]$ & $\begin{array}{lr}\text { SLICOTS } & \text { (Rule } \\
\text { based } & \text { DDoS } \\
\text { mitigation) } & \end{array}$ & $\begin{array}{l}\text { TCP-SYN flooding } \\
\text { attacks }\end{array}$ & Memory & $\begin{array}{l}\text { OpenDay- } \\
\text { light, } \\
\text { Mininet }\end{array}$ & Single & $\begin{array}{l}\text { Smart Switches, switch can } \\
\text { take decisions }\end{array}$ & $\begin{array}{l}\text { Functioning only when con- } \\
\text { troller behaves in reactive } \\
\text { manner, may increase con- } \\
\text { trol plane bandwidth con- } \\
\text { sumption and overload }\end{array}$ \\
\hline
\end{tabular}




\begin{tabular}{|c|c|c|c|c|c|c|c|}
\hline Ref. & Defense mechanism & Intrusion type & $\begin{array}{l}\text { Targeted } \\
\text { resources }\end{array}$ & $\begin{array}{l}\text { Simulation } \\
\text { tools }\end{array}$ & Controller & Advantages & Limitations \\
\hline [75] & Avant-guard & TCP-SYN flooding & Memory & POX & Single & $\begin{array}{l}\text { Improves resilience against } \\
\text { TCP SYN flood and network } \\
\text { scanning attacks }\end{array}$ & $\begin{array}{l}\text { Restricted to the number } \\
\text { of proxy ports, not easily } \\
\text { deployable in practice, in- } \\
\text { valid to non-TCP protocols } \\
\text { based attacks }\end{array}$ \\
\hline$[76]$ & $\begin{array}{lr}\text { OverWatch } & (\text { au- } \\
\text { toencoder } & \text { based } \\
\text { detection }) & \end{array}$ & $\begin{array}{l}\text { Flooding attacks } \\
(\mathrm{SYN}, \mathrm{UDP}, \mathrm{ICMP})\end{array}$ & $\begin{array}{l}\text { Bandwidth, } \\
\text { memory }\end{array}$ & $\begin{array}{l}\text { Ryu, Real } \\
\text { time }\end{array}$ & Single & $\begin{array}{l}\text { Rapid reaction to threats re- } \\
\text { duces southbound interface } \\
\text { overhead }\end{array}$ & 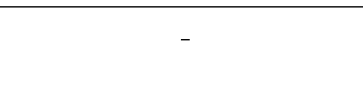 \\
\hline$[77]$ & $\begin{array}{l}\text { SDNScore ( statis- } \\
\text { tical and packet } \\
\text { based defense mech- } \\
\text { anism) }\end{array}$ & $\begin{array}{l}\text { TCP-SYN, DNS, } \\
\text { SQL Slammer, } \\
\text { NTP attacks }\end{array}$ & $\begin{array}{l}\text { Memory, } \\
\text { CPU }\end{array}$ & $\begin{array}{l}\text { Discrete } \\
\text { event sim- } \\
\text { ulator } \\
\text { (name not } \\
\text { mentioned) }\end{array}$ & Single & $\begin{array}{l}\text { Can detect also new unknown } \\
\text { attacks }\end{array}$ & $\begin{array}{l}\text { Per-packet processing over- } \\
\text { head }\end{array}$ \\
\hline [78] & $\begin{array}{l}\text { StateSec (entropy } \\
\text { based algorithm } \\
\text { with such monitor- } \\
\text { ing features (IP and } \\
\text { port addresses)) }\end{array}$ & $\begin{array}{l}\text { DoS/DDoS flood- } \\
\text { ing and port scan }\end{array}$ & Bandwidth & $\begin{array}{l}\text { Ryu, OVS, } \\
\text { Mininet }\end{array}$ & Single & $\begin{array}{l}\text { Improves the reaction time to } \\
\text { threats, reduces burden of the } \\
\text { controller and control channel, } \\
\text { also extended to detect slow } \\
\text { DDoS [84] }\end{array}$ & $\begin{array}{l}\text { Per-packet processing over- } \\
\text { head }\end{array}$ \\
\hline$[80]$ & SDN-Guard & $\begin{array}{l}\text { DoS flooding (TCP- } \\
\text { SYN, UDP, ICMP) }\end{array}$ & $\begin{array}{l}\text { Bandwidth, } \\
\text { memory }\end{array}$ & $\begin{array}{l}\text { Floodlight, } \\
\text { Mininet }\end{array}$ & Single & $\begin{array}{l}\text { Less deployment time, reduces } \\
\text { switch to IDS traffic }\end{array}$ & $\begin{array}{l}\text { Complex and processing } \\
\text { overhead }\end{array}$ \\
\hline$[83]$ & $\begin{array}{l}\text { Flood-guard (based } \\
\text { on proactive flow } \\
\text { rule analyzer and } \\
\text { packet migration } \\
\text { for mitigation) }\end{array}$ & $\begin{array}{l}\text { DoS flooding (UDP, } \\
\text { TCP, ICMP) }\end{array}$ & $\begin{array}{l}\text { Bandwidth, } \\
\text { memory }\end{array}$ & $\begin{array}{l}\text { POX, } \\
\text { Mininet } \\
\text { (Software } \\
\text { and hard- } \\
\text { ware both } \\
\text { types of } \\
\text { evaluation) }\end{array}$ & Single & $\begin{array}{l}\text { Modifiactions are not required } \\
\text { in the existing SDN infrastruc- } \\
\text { ture }\end{array}$ & $\begin{array}{l}\text { Lacks portability in net- } \\
\text { work deployment, may not } \\
\text { ensure fairness of non- } \\
\text { attack traffic }\end{array}$ \\
\hline [73] & 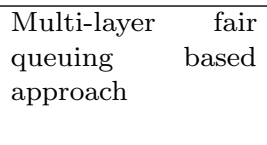 & UDP flooding & $\begin{array}{l}\text { Memory, } \\
\text { CPU }\end{array}$ & $\begin{array}{l}\text { Floodlight, } \\
\text { OVS, } \\
\text { Mininet } \\
\text { (testbed) }\end{array}$ & Single & $\begin{array}{l}\text { Modifications and extra appli- } \\
\text { ances are not required at data } \\
\text { plane }\end{array}$ & $\begin{array}{l}\text { Applicable for a specific } \\
\text { type DDoS }\end{array}$ \\
\hline
\end{tabular}




\subsection{Trade-off between concepts of actual SDN and smart switches}

SDN switches are not capable to take any smart decision from their own in an unobvious situation. This property leads to sending huge volume of unknown traffic to the controller that can create communication overhead. Therefore, some researches have suggested providing intelligence to SDN switches for enabling it to take some decisive actions. This feature can reduce the burden on the controller and its chances to get collapsed. However, the main fundamental concept of the SDN having simple forwarding switches should not be compromised. Therefore, the system designer should be careful for this trade-off between the actual SDN concept and the smarter switches.

\subsection{Slow DDoS}

It is very tough to discover slow and low rate DDoS attacks because traffic flows in slow attacks act just like benign traffic flows. It requires very less resources to get launched and even can make unavailable the services of the web servers using just one host. High rate DDoS are more easier in a way than slow DDoS. Hence, slow DDoS attack mitigation needs some serious efforts and research work.

\subsection{Lack of standard communication protocols and harmful applications}

For the communication of applications and control plane, there is no standard northbound interface used yet. Northbound interface provides a programmable nature to install the security and other required applications into the control plane. This open and programmable nature can make it vulnerable to malicious applications that can even change the complete network functionality and provide unexpected results. Attackers can implement their own policies and add to the controller to take the control of network in their hands. This insecure application-control channel may be a convenient target for the attackers, hence securing the communication channel becomes an important issue.

The flexibility and programmability are the key features of SDN. These features expose the network to the user applications. This may result in installation of malicious applications with fake rules. The malicious applications can degrade the performance of the controller. Protecting the controller from malicious applications can be considered as an another research area.

\subsection{Scalability and interoperability of controllers}

Controller is the most salient part of SDN. As network size increases, single controller is not capable to handle all the traffic alone. Therefore, backup and additional controllers are deployed to reduce the chance of single point of failure and to handle the traffic. However, different controllers have different policies and routing techniques. Distributed controllers suffer from scalability and interoperability. Interoperability of controllers being used in different networks for facilitating consistent network operation and scalability needs attention. An standard east-west bound interface is recommended for secure communication between controllers.

\subsection{Efficient analysis of network traffic}

DDoS defense techniques need real-time monitoring and tracing of the network traffic to be analyzed. Use of tools (sFlow, netFlow, etc.) to monitor the traffic may cause additional overhead. The detection mechanisms may utilize packet-based or flow-based traffic analysis. The packet-based analysis imposes large overhead while increasing the accuracy while less 
overhead with low accuracy is achieved in flow-based analysis. Hence, finding the best trade-off between overhead and accuracy can also be considered as an important research issue.

\subsection{A Sole solution for all DDoS}

A defense mechanism should be able to mitigate different kinds of DDoS attacks. Existing DDoS defense mechanisms found in literature can handle only a specific type of DDoS attack. These mechanisms are designed with a restricted hardware appliance and a fix functionality that are incapable of handling different kinds of attacks. These security solutions need to be enhanced to detect more types of attacks with minimum communication overhead. Thus developing a mechanism to defend multiple DDoS attacks is a major research issue.

\subsection{Ability to analyze real DDoS}

In most of the existing defense solutions, a small network scenario consisting of few devices is used to test the performance of the solutions. Hence, the performance of these solutions may not work well in the case of large networks. Such small networking environment may not be able to demonstrate the defense of real DDoS attacks completely. This issue should be considered as a major research challenge. Therefore, there is a need to analyze the real attacks defense by providing scalability in various large network scenarios. The defense mechanisms should be designed in a way to be able to analyze real attack cases.

\section{OUR RESEARCH CONTRIBUTION AND ITS SIGNIFICANCE}

In this research paper, a detailed study on SDN security issues is done. There are few research articles that are available in literature related to DDoS attacks and SDN security. Wang et al. [85] discussed various security issues in the cloud environment in 2015 that can be handled by flexible SDN paradigm. For securing cloud, Yan et al. [86] have discussed the DDoS and SDN security. One existing work [87] has described the DDoS vulnerabilities, detection and mitigation mechanisms by using centralized characteristic of SDN. They divided the discussed mechanisms on the basis of used detection methods such as based of entropy, ML, and traffic analysis. Further, the mitigation methods such as dropping the packets or blocking the port are also studied. However, the authors have not studied DDoS attacks for the SDN security solutions. Kalkan et al. [88] have presented DDoS defense solutions in the SDN environment and classified these solutions on the basis of detection methods. Imran et al. [89] provided various defense mechanisms against DoS attacks and classified them according to their strategies to reduce the impact of attacks. Authors have also presented some limitations of existing defensive mechanisms.

The motivation behind this research work is to highlight the present security challenges and their countermeasures in the domain of SDN based DDoS defense mechanisms. It covers most of the possible issues altogether that must be considered for effective security of SDN based networks. The SDN and DDoS have an antithetical relationship with each other. On one side, SDN can help in defeating DDoS attacks by utilizing its security features. On the other side, SDN itself becomes a target of attackers because of its inherent design issues. As far as our literature is concerned, this antithetical correspondence of DDoS and SDN security solutions is not studied deeply in earlier research. This work contains SDN specific DDoS threats and its impact on the SDN architecture. The paper covers all the potential DDoS based vulnerabilities that can cause harm to SDN. It is also observed that SDN can be collaborated with some new technologies such as NFV, Blockchain, Smart contracts, IoT, and Honeynet etc. This collaboration of technologies can improve the existing mitigation

ACM Comput. Surv., Vol. 1, No. 1, Article 1. Publication date: January 2018. 
systems. Such approaches can provide the adaptability and dynamic functionality to the cloud vendors and their customers.

\section{NEW RESEARCH DIRECTIONS}

A significant research direction can also be combining SDN with information-centric networks (ICN). ICN has come to the fore as the traditional networking paradigms are host-centric whereas the user's main focus is on getting access to the information regardless of from where it comes. To support this concept, ICN [90] extracts the information or the content from the IP packets. ICN [91] offers named-based routing and in-network caching to the modern networks. It provides fine grained control on the information transfer. The integration of ICN with SDN provides better content-centric security due to adaptive nature of SDN and inherent working of ICN. ICN has direct access to content as it is able to do named based caching of content at intermediate network nodes. SDN can obtain such content available in the packets directly. The transmission of named content instead of full IP packet reduces the network overhead and increases the throughput. The integration of SDN and ICN improves management of the network as well as providing security services to each other [92]. ICN prevents the data plane from different threats by offering self-certifying names and content based security mechanisms. ICN reduces the probability of spoofing and interception of communication on switch-controller bandwidth by giving some authenticated control messages. One important security benefit of ICN is that it focuses on content rather than location or IP address so that the controller cannot be impersonated by some malicious/fake entity. Therefore, the integration of ICN and SDN should offer better and efficient network services.

A new research direction can be enhancement of security in Bring Your Own Computer (BYOD) policy using SDN. According to BYOD policy, employees are free to use their own mobile devices and gadgets to access the workplace's services, which is going to become a trend soon. Centralized control of SDN may keep an eye at all the activities happening in BYOD environment for the security perspective. SDN makes it possible to quickly deployment of security services to provide more security to BYOD organizations.

It is already known that SDN suffers from various security issues due to centralized and open programmable behavior. Therefore, SDN controller should incorporate network behavior measurement modules for its security. For early detection of anomalies and malicious activities integration of measurement tools and SDN can be considered as a new research direction. Some important network state measuring parameters could be network latency, available bandwidth, and topology discovery. The measurement tools for these parameters can be deployed in the controller to monitor all the states in the network periodically. The analyzed network behavior may provide advance information against imminent threats.

A new research direction is fusion of SDN and traditional/legacy networks. Due to high cost of SDN devices, it may not be economically feasible to set up a pure SDN network. A unified network using SDN and non SDN technologies may offer benefits of SDN for easy deployment of security services. As SDN attracts attacks such as DDoS, code injection, man-in-the middle etc., efficient defense mechanisms are needed to secure the network However, security of such networks that use both SDN and legacy network technologies is an important open research direction. 
Table 4. Analysis of defense mechanisms for SDN

\begin{tabular}{|c|c|c|c|c|c|}
\hline Authors/Ref. & Cause of attack & Defense approach & $\begin{array}{l}\text { Switch } \\
\text { over- } \\
\text { load }\end{array}$ & $\begin{array}{l}\text { Control } \\
\text { plane } \\
\text { bandwidth } \\
\text { congestion }\end{array}$ & $\begin{array}{l}\text { Controller } \\
\text { satura- } \\
\text { tion }\end{array}$ \\
\hline $\begin{array}{lll}\text { Wu } & \text { et } & \text { al. } \\
{[82]} & & \end{array}$ & Centralized control & $\begin{array}{l}\text { By flow migration be- } \\
\text { tween two controllers }\end{array}$ & - & Yes & Yes \\
\hline $\begin{array}{l}\text { Han et al. } \\
{[76]}\end{array}$ & Dumb switches & $\begin{array}{l}\text { By using collaborative } \\
\text { intelligence between } \\
\text { switch \& controller }\end{array}$ & - & Yes & - \\
\hline $\begin{array}{l}\text { Wang et al. } \\
{[71]}\end{array}$ & $\begin{array}{l}\text { Single point \& cas- } \\
\text { cading failure of con- } \\
\text { trollers }\end{array}$ & $\begin{array}{l}\text { By bandwidth predic- } \\
\text { tion \& controller dy- } \\
\text { namic scheduling }\end{array}$ & - & - & Yes \\
\hline Xu et al. [66] & $\begin{array}{lll}\text { Limited TCAM in } \\
\text { switches }\end{array}$ & $\begin{array}{l}\text { Using statistical and to- } \\
\text { ken bucket approach }\end{array}$ & Yes & - & - \\
\hline $\begin{array}{l}\text { Durner et al. } \\
{[68]}\end{array}$ & $\begin{array}{lll}\text { Limited TCAM in } \\
\text { switches }\end{array}$ & $\begin{array}{l}\text { By using statistical ap- } \\
\text { proach and hashing } \\
\text { function }\end{array}$ & Yes & - & - \\
\hline $\begin{array}{l}\text { Mohammadi } \\
\text { et al. [74] }\end{array}$ & Separation of planes & $\begin{array}{l}\text { By using dynamic pro- } \\
\text { grammability nature of } \\
\text { SDN }\end{array}$ & - & Yes & - \\
\hline $\begin{array}{l}\text { Kalkan et al. } \\
{[77]}\end{array}$ & Dumb switches & $\begin{array}{l}\text { By making switches } \\
\text { smarter to take actions }\end{array}$ & - & Yes & Yes \\
\hline $\begin{array}{l}\text { Boite et al. } \\
{[78]}\end{array}$ & Dumb switches & $\begin{array}{l}\text { By delegating local pro- } \\
\text { cessing to switches }\end{array}$ & - & Yes & Yes \\
\hline $\begin{array}{l}\text { Dridi et al. } \\
{[80]}\end{array}$ & $\begin{array}{l}\text { Central control of } \\
\text { SDN network }\end{array}$ & $\begin{array}{l}\text { By leveraging an IDS } \\
\text { and finding an optimal } \\
\text { placement for IDS }\end{array}$ & Yes & Yes & Yes \\
\hline $\begin{array}{l}\text { Macedo et } \\
\text { al. }[72]\end{array}$ & Single point of failure & $\begin{array}{l}\text { Through clustering of } \\
\text { the controllers }\end{array}$ & - & - & Yes \\
\hline $\begin{array}{l}\text { Mousavi et } \\
\text { al. [69] }\end{array}$ & Single point of failure & $\begin{array}{l}\text { Variation in entropy of } \\
\text { destination IP address }\end{array}$ & - & - & Yes \\
\hline $\begin{array}{l}\text { Wang et al. } \\
{[83]}\end{array}$ & $\begin{array}{l}\text { Due to large amount } \\
\text { of table-miss mes- } \\
\text { sages in switches }\end{array}$ & $\begin{array}{l}\text { By proactive flow rule } \\
\text { analyzer and packet mi- } \\
\text { gration }\end{array}$ & - & Yes & - \\
\hline $\begin{array}{l}\text { Shin et al. } \\
{[75]}\end{array}$ & Separation of planes & $\begin{array}{l}\text { Based on connection } \\
\text { migration (inspired by } \\
\text { SYN proxy) }\end{array}$ & - & Yes & - \\
\hline
\end{tabular}

\section{CONCLUSION}

This work is significantly centered around the recent advancements and progressions in detection and mitigation procedures for defending SDN security from DDoS. Two prospectives of SDN security are considered. In first, SDN may help to protect the traditional networks while in second SDN may be a victim itself. Various defense mechanisms are classified into two categories i.e., defense by SDN and defense for SDN that are based on the design characteristics of the SDN architecture. Further, a comparison of these mechanisms has been discussed on the basis of the detection and mitigation algorithms. It is concluded that there has been a significant growth in the research field of providing security by utilizing the SDN 
features. However, from the viewpoint of the impacts of DDoS threats in SDN, it can be a DDoS target itself because of its centralized nature. SDN is not completely secure hence, there is a need to explore more efficient defense mechanisms for DDoS mitigation.

\section{REFERENCES}

[1] Nick Feamster, Jennifer Rexford, and Ellen Zegura. The road to sdn: an intellectual history of programmable networks. ACM SIGCOMM Computer Communication Review, 44(2):87-98, 2014.

[2] Akram Hakiri, Aniruddha Gokhale, Pascal Berthou, Douglas C. Schmidt, and Thierry Gayraud. Softwaredefined networking: Challenges and research opportunities for future internet. Computer Networks, $75: 453-471,2014$.

[3] D. Kreutz, F. M. V. Ramos, P. E. Veríssimo, C. E. Rothenberg, S. Azodolmolky, and S. Uhlig. Softwaredefined networking: A comprehensive survey. Proceedings of the IEEE, 103(1):14-76, Jan 2015.

[4] J. Tourrilhes, P. Sharma, S. Banerjee, and J. Pettit. Sdn and openflow evolution: A standards perspective. Computer, 47(11):22-29, Nov 2014.

[5] Paul Goransson, Chuck Black, and Timothy Culver. Software defined networks: a comprehensive approach. Morgan Kaufmann, 2016.

[6] Keith Kirkpatrick. Software-defined networking. Communications of the ACM, 56(9):16-19, 2013.

[7] H. Kim and N. Feamster. Improving network management with software defined networking. IEEE Communications Magazine, 51(2):114-119, February 2013.

[8] Per Oscarson. Information security fundamentals. In Security education and critical infrastructures, pages 95-107. Springer, 2003.

[9] Muhammad Umar Farooq, Muhammad Waseem, Anjum Khairi, and Sadia Mazhar. A critical analysis on the security concerns of internet of things (iot). International Journal of Computer Applications, 111(7), 2015.

[10] Christos Douligeris and Aikaterini Mitrokotsa. Ddos attacks and defense mechanisms: classification and state-of-the-art. Computer Networks, 44(5):643-666, 2004.

[11] Stephen M Specht and Ruby B Lee. Distributed denial of service: Taxonomies of attacks, tools, and countermeasures. In ISCA PDCS, pages 543-550, 2004.

[12] A. D. Wood and J. A. Stankovic. Denial of service in sensor networks. Computer, 35(10):54-62, Oct 2002.

[13] Dan Goodin. Us service provider survives the biggest recorded ddos in history, mar 2018.

[14] Rob Enns. Netconf configuration protocol. 2006.

[15] Xianfeng Li and Wencong Xie. Craft: A cache reduction architecture for flow tables in software-defined networks. In 2017 IEEE Symposium on Computers and Communications (ISCC), pages 967-972, July 2017.

[16] Naga Katta, Omid Alipourfard, Jennifer Rexford, and David Walker. Cacheflow: Dependency-aware rule-caching for software-defined networks. In Proceedings of the Symposium on SDN Research, SOSR '16, pages 6:1-6:12, New York, NY, USA, 2016. ACM.

[17] Giuseppe Bianchi, Marco Bonola, Antonio Capone, and Carmelo Cascone. Openstate: Programming platform-independent stateful openflow applications inside the switch. SIGCOMM Comput. Commun. Rev., 44(2):44-51, April 2014.

[18] CALYPTIX. Ddos attacks 101: Types, targets, and motivations, April 2015.

[19] B. B. Gupta, R. C. Joshi, and Manoj Misra. Defending against distributed denial of service attacks: Issues and challenges. Information Security Journal: A Global Perspective, 18(5):224-247, 2009.

[20] N Muraleedharan and B Janet. Behaviour analysis of http based slow denial of service attack. In Wireless Communications, Signal Processing and Networking (WiSPNET), 2017 International Conference on, pages 1851-1856. IEEE, 2017.

[21] BB Gupta and Omkar P Badve. Taxonomy of dos and ddos attacks and desirable defense mechanism in a cloud computing environment. Neural Computing and Applications, 28(12):3655-3682, 2017.

[22] L. Feinstein, D. Schnackenberg, R. Balupari, and D. Kindred. Statistical approaches to ddos attack detection and response. In Proceedings DARPA Information Survivability Conference and Exposition, volume 1, pages 303-314 vol.1, April 2003.

[23] D. Sattar, A. Matrawy, and O. Adeojo. Adaptive bubble burst (abb): Mitigating ddos attacks in software-defined networks. In 2016 17th International Telecommunications Network Strategy and Planning Symposium (Networks), pages 50-55, Sept 2016. 
[24] Ahmad Ariff Aizuddin, Mohd Atan, Megat Norulazmi, Megat Mohamed Noor, Shadil Akimi, and Zainal Abidin. Dns amplification attack detection and mitigation via sflow with security-centric sdn. In Proceedings of the 11th International Conference on Ubiquitous Information Management and Communication, IMCOM '17, pages 3:1-3:7, New York, NY, USA, 2017. ACM.

[25] Sonia Panchen, Peter Phaal, and Neil McKee. Inmon corporation's sflow: A method for monitoring traffic in switched and routed networks. 2001

[26] Jiaqi Yan and Dong Jin. Vt-mininet: Virtual-time-enabled mininet for scalable and accurate softwaredefine network emulation. In Proceedings of the 1st ACM SIGCOMM Symposium on Software Defined Networking Research, SOSR '15, pages 27:1-27:7, New York, NY, USA, 2015. ACM.

[27] Ben Pfaff, Justin Pettit, Teemu Koponen, Ethan J Jackson, Andy Zhou, Jarno Rajahalme, Jesse Gross, Alex Wang, Joe Stringer, Pravin Shelar, et al. The design and implementation of open vswitch. In NSDI, pages 117-130, 2015.

[28] Christian Rossow. Amplification hell: Revisiting network protocols for ddos abuse. In NDSS, 2014.

[29] David Huistra. Detecting reflection attacks in dns flows. 2013.

[30] Q. Yan, Q. Gong, and F. R. Yu. Effective software-defined networking controller scheduling method to mitigate ddos attacks. Electronics Letters, 53(7):469-471, 2017.

[31] Ligia Rodrigues Prete, A. A. Shinoda, C. M. Schweitzer, and R. L. S. de Oliveira. Simulation in an sdn network scenario using the pox controller. In 2014 IEEE Colombian Conference on Communications and Computing (COLCOM), pages 1-6, June 2014.

[32] Ming-Hung Chen, Jyun-Yan Ciou, I-Hsin Chung, and Cheng-Fu Chou. Flexprotect: A sdn-based ddos attack protection architecture for multi-tenant data centers. In Proceedings of the International Conference on High Performance Computing in Asia-Pacific Region, HPC Asia 2018, pages 202-209, New York, NY, USA, 2018. ACM.

[33] J. Zheng, Q. Li, G. Gu, J. Cao, D. K. Y. Yau, and J. Wu. Realtime ddos defense using cots sdn switches via adaptive correlation analysis. IEEE Transactions on Information Forensics and Security, 13(7):1838-1853, July 2018.

[34] C. Buragohain and N. Medhi. Flowtrapp: An sdn based architecture for ddos attack detection and mitigation in data centers. In 2016 3rd International Conference on Signal Processing and Integrated Networks (SPIN), pages 519-524, Feb 2016.

[35] K. Hong, Y. Kim, H. Choi, and J. Park. Sdn-assisted slow http ddos attack defense method. IEEE Communications Letters, PP(99):1-1, Aug 2017.

[36] Mark Shtern, Roni Sandel, Marin Litoiu, Chris Bachalo, and Vasileios Theodorou. Towards mitigation of low and slow application ddos attacks. In Cloud Engineering (IC2E), 2014 IEEE International Conference on, pages 604-609. IEEE, 2014.

[37] Thomas Lukaseder, Lisa Maile, Benjamin Erb, and Frank Kargl. Sdn-assisted network-based mitigation of slow ddos attacks. arXiv preprint arXiv:1804.06750, 2018.

[38] Nikhil Tripathi, Neminath Hubballi, and Yogendra Singh. How secure are web servers? an empirical study of slow http dos attacks and detection. In Availability, Reliability and Security (ARES), 2016 11th International Conference on, pages 454-463. IEEE, 2016.

[39] Tetsuya Hirakawa, Kanayo Ogura, Bhed Bahadur Bista, and Toyoo Takata. A defense method against distributed slow http dos attack. In Network-Based Information Systems (NBiS), 2016 19th International Conference on, pages 152-158. IEEE, 2016.

[40] Clifford Kemp, Chad Calvert, and Taghi Khoshgoftaar. Utilizing netflow data to detect slow read attacks. In 2018 IEEE International Conference on Information Reuse and Integration (IRI), pages 108-116. IEEE, 2018.

[41] Truong Thu Huong and Nguyen Huu Thanh. Software defined networking-based one-packet ddos mitigation architecture. In Proceedings of the 11th International Conference on Ubiquitous Information Management and Communication, IMCOM '17, pages 110:1-110:7, New York, NY, USA, 2017. ACM.

[42] Rishikesh Sahay, Gregory Blanc, Zonghua Zhang, Khalifa Toumi, and Hervé Debar. Adaptive policydriven attack mitigation in sdn. In Proceedings of the 1st International Workshop on Security and Dependability of Multi-Domain Infrastructures, XDOMO'17, pages 4:1-4:6, New York, NY, USA, 2017. ACM.

[43] Rishikesh Sahay, Gregory Blanc, Zonghua Zhang, and Hervé Debar. Aroma: An sdn based autonomic ddos mitigation framework. Computers and Security, 70:482 - 499, 2017.

[44] Sufian Hameed and Hassan Ahmed Khan. Sdn based collaborative scheme for mitigation of ddos attacks. Future Internet, 10(3), 2018. 
[45] Lei Wang, Qing Li, Yong Jiang, Xuya Jia, and Jianping Wu. Woodpecker: Detecting and mitigating link-flooding attacks via sdn. Computer Networks, 147:1-13, 2018.

[46] Hojjat Adeli and Shih-Lin Hung. Machine Learning: Neural Networks, Genetic Algorithms, and Fuzzy Systems. John Wiley \& Sons, Inc., New York, NY, USA, 1994.

[47] Ioannis Tsochantaridis, Thomas Hofmann, Thorsten Joachims, and Yasemin Altun. Support vector machine learning for interdependent and structured output spaces. In Proceedings of the Twenty-first International Conference on Machine Learning, ICML '04, pages 104-, New York, NY, USA, 2004 ACM.

[48] Teuvo Kohonen. The self-organizing map. Neurocomputing, 21(1):1 - 6, 1998.

[49] J. Ashraf and S. Latif. Handling intrusion and ddos attacks in software defined networks using machine learning techniques. In 2014 National Software Engineering Conference, pages 55-60, Nov 2014.

[50] Quamar, Weiqing Sun, and Ahmad Y. Javaid. A deep learning based ddos detection system in software-defined networking (SDN). CoRR, abs/1611.07400, 2016.

[51] Chuanhuang Li, Yan Wu, Xiaoyong Yuan, Zhengjun Sun, Weiming Wang, Xiaolin Li, and Liang Gong. Detection and defense of ddos attack-based on deep learning in openflow-based sdn. International Journal of Communication Systems, 31(5):e3497, 2018.

[52] M. E. Ahmed, H. Kim, and M. Park. Mitigating dns query-based ddos attacks with machine learning on software-defined networking. In MILCOM 2017 - 2017 IEEE Military Communications Conference (MILCOM), pages 11-16, Oct 2017.

[53] D. Hu, P. Hong, and Y. Chen. Fadm: Ddos flooding attack detection and mitigation system in softwaredefined networking. In GLOBECOM 2017 - 2017 IEEE Global Communications Conference, pages 1-7, Dec 2017.

[54] Yunhe Cui, Lianshan Yan, Saifei Li, Huanlai Xing, Wei Pan, Jian Zhu, and Xiaoyang Zheng. Sd-antiddos: Fast and efficient ddos defense in software-defined networks. Journal of Network and Computer Applications, 68:65 - 79, 2016.

[55] Mohd Zafran Abdul Aziz and Koji Okamura. Leveraging sdn for detection and mitigation smtp flood attack through deep learning analysis techniques. IJCSNS, 17(10):166, 2017.

[56] A. Santos da Silva, J. A. Wickboldt, L. Z. Granville, and A. Schaeffer-Filho. Atlantic: A framework for anomaly traffic detection, classification, and mitigation in sdn. In NOMS 2016 - 2016 IEEE/IFIP Network Operations and Management Symposium, pages 27-35, April 2016.

[57] Jin Ye, Xiangyang Cheng, Jian Zhu, Luting Feng, and Ling Song. A ddos attack detection method based on svm in software defined network. Security and Communication Networks, 2018, 2018.

[58] S. Lee, J. Kim, S. Shin, P. Porras, and V. Yegneswaran. Athena: A framework for scalable anomaly detection in software-defined networks. In 2017 47th Annual IEEE/IFIP International Conference on Dependable Systems and Networks (DSN), pages 249-260, June 2017.

[59] M. V. O. De Assis, A. H. Hamamoto, T. Abrão, and M. L. Proença. A game theoretical based system using holt-winters and genetic algorithm with fuzzy logic for dos/ddos mitigation on sdn networks IEEE Access, 5:9485-9496, 2017.

[60] C. C. Chen, Y. R. Chen, W. C. Lu, S. C. Tsai, and M. C. Yang. Detecting amplification attacks with software defined networking. In 2017 IEEE Conference on Dependable and Secure Computing, pages 195-201, Aug 2017.

[61] Pankaj Berde, Matteo Gerola, Jonathan Hart, Yuta Higuchi, Masayoshi Kobayashi, Toshio Koide, Bob Lantz, Brian O'Connor, Pavlin Radoslavov, William Snow, et al. Onos: towards an open, distributed sdn os. In Proceedings of the third workshop on Hot topics in software defined networking, pages 1-6. ACM, 2014.

[62] Qiao Yan and Wenyao Huang. A ddos detection and mitigation system framework based on spark and sdn. In Meikang Qiu, editor, Smart Computing and Communication, pages 350-358, Cham, 2017. Springer International Publishing.

[63] D. He, S. Chan, X. Ni, and M. Guizani. Software-defined-networking-enabled traffic anomaly detection and mitigation. IEEE Internet of Things Journal, 4(6):1890-1898, Dec 2017.

[64] Adel Alshamrani, Ankur Chowdhary, Sandeep Pisharody, Duo Lu, and Dijiang Huang. A defense system for defeating ddos attacks in sdn based networks. In Proceedings of the 15th ACM International Symposium on Mobility Management and Wireless Access, MobiWac '17, pages 83-92, New York, NY, USA, 2017. ACM.

[65] Jing Liu, Yingxu Lai, and Shixuan Zhang. Fl-guard: A detection and defense system for ddos attack in sdn. In Proceedings of the 2017 International Conference on Cryptography, Security and Privacy, ICCSP '17, pages 107-111, New York, NY, USA, 2017. ACM. 
[66] T. Xu, D. Gao, P. Dong, C. H. Foh, and H. Zhang. Mitigating the table-overflow attack in softwaredefined networking. IEEE Transactions on Network and Service Management, 14(4):1086-1097, Dec 2017.

[67] Z. K. Khattak, M. Awais, and A. Iqbal. Performance evaluation of opendaylight sdn controller. In 2014 20th IEEE International Conference on Parallel and Distributed Systems (ICPADS), pages 671-676, Dec 2014.

[68] R. Durner, C. Lorenz, M. Wiedemann, and W. Kellerer. Detecting and mitigating denial of service attacks against the data plane in software defined networks. In 2017 IEEE Conference on Network Softwarization (NetSoft), pages 1-6, July 2017.

[69] S. M. Mousavi and M. St-Hilaire. Early detection of ddos attacks against sdn controllers. In 2015 International Conference on Computing, Networking and Communications (ICNC), pages 77-81, Feb 2015.

[70] Kshira Sagar Sahoo, Deepak Puthal, Mayank Tiwary, Joel JPC Rodrigues, Bibhudatta Sahoo, and Ratnakar Dash. An early detection of low rate ddos attack to sdn based data center networks using information distance metrics. Future Generation Computer Systems, 89:685-697, 2018.

[71] Tao Wang, Hongchang Chen, Guozhen Cheng, and Yulin Lu. Sdnmanager: A safeguard architecture for sdn dos attacks based on bandwidth prediction. Security and Communication Networks, 2018, 2018.

[72] R. Macedo, R. de Castro, A. Santos, Y. Ghamri-Doudane, and M. Nogueira. Self-organized sdn controller cluster conformations against ddos attacks effects. In 2016 IEEE Global Communications Conference (GLOBECOM), pages 1-6, Dec 2016.

[73] Peng Zhang, Huanzhao Wang, Chengchen Hu, and Chuang Lin. On denial of service attacks in software defined networks. IEEE Network, 30(6):28-33, 2016.

[74] R. Mohammadi, R. Javidan, and M. Conti. Slicots: An sdn-based lightweight countermeasure for tcp syn flooding attacks. IEEE Transactions on Network and Service Management, 14(2):487-497, June 2017.

[75] Seungwon Shin, Vinod Yegneswaran, Phillip A. Porras, and Guofei Gu. Avant-guard: scalable and vigilant switch flow management in software-defined networks. In ACM Conference on Computer and Communications Security, 2013.

[76] Biao Han, Xiangrui Yang, Zhigang Sun, Jinfeng Huang, and Jinshu Su. Overwatch: A cross-plane ddos attack defense framework with collaborative intelligence in sdn. Security and Communication Networks 2018, 2018.

[77] K. Kalkan, G. Gür, and F. Alagöz. Sdnscore: A statistical defense mechanism against ddos attacks in sdn environment. In 2017 IEEE Symposium on Computers and Communications (ISCC), pages 669-675, July 2017.

[78] J. Boite, P. A. Nardin, F. Rebecchi, M. Bouet, and V. Conan. Statesec: Stateful monitoring for ddos protection in software defined networks. In 2017 IEEE Conference on Network Softwarization (NetSoft), pages $1-9$, July 2017.

[79] Giuseppe Bianchi, Marco Bonola, Antonio Capone, and Carmelo Cascone. Openstate: programming platform-independent stateful openflow applications inside the switch. ACM SIGCOMM Computer Communication Review, 44(2):44-51, 2014.

[80] Lobna Dridi and Mohamed Faten Zhani. A holistic approach to mitigating dos attacks in sdn networks. International Journal of Network Management, 2017.

[81] Zhuo Chen, Fu Jiang, Yijun Cheng, Xin Gu, Weirong Liu, and Jun Peng. Xgboost classifier for ddos attack detection and analysis in sdn-based cloud. In Big Data and Smart Computing (BigComp), 2018 IEEE International Conference on, pages 251-256. IEEE, 2018.

[82] Pengpeng Wu, Lin Yao, Chi Lin, Guowei Wu, and Mohammad S. Obaidat. Fmd: A dos mitigation scheme based on flow migration in software-defined-networking. International Journal of Communication Systems, 0(0):e3543, 2018.

[83] H. Wang, L. Xu, and G. Gu. Floodguard: A dos attack prevention extension in software-defined networks. In 2015 45th Annual IEEE/IFIP International Conference on Dependable Systems and Networks, pages $239-250,2015$.

[84] F. Rebecchi, J. Boite, P. A. Nardin, M. Bouet, and V. Conan. Traffic monitoring and ddos detection using stateful sdn. In 2017 IEEE Conference on Network Softwarization (NetSoft), pages 1-2, July 2017.

[85] Bing Wang, Yao Zheng, Wenjing Lou, and Y. Thomas Hou. Ddos attack protection in the era of cloud computing and software-defined networking. Computer Networks, 81:308 - 319, 2015. 
[86] Q. Yan, F. R. Yu, Q. Gong, and J. Li. Software-defined networking (sdn) and distributed denial of service (ddos) attacks in cloud computing environments: A survey, some research issues, and challenges. IEEE Communications Surveys Tutorials, 18(1):602-622, Firstquarter 2016.

[87] Narmeen Zakaria Bawany, Jawwad A. Shamsi, and Khaled Salah. Ddos attack detection and mitigation using sdn: Methods, practices, and solutions. Arabian Journal for Science and Engineering, 42(2):425441, Feb 2017.

[88] K. Kalkan, G. Gur, and F. Alagoz. Defense mechanisms against ddos attacks in sdn environment. IEEE Communications Magazine, 55(9):175-179, 2017.

[89] Muhammad Imran, Muhammad Hanif Durad, Farrukh Aslam Khan, and Abdelouahid Derhab. Toward an optimal solution against denial of service attacks in software defined networks. Future Generation Computer Systems, 92:444-453, 2019.

[90] Muhammad Azfar Yaqub, Syed Hassan Ahmed, Safdar Hussain Bouk, and Dongkyun Kim. Informationcentric networks (icn). In Content-Centric Networks, pages 19-33. Springer, 2016.

[91] Bengt Ahlgren, Christian Dannewitz, Claudio Imbrenda, Dirk Kutscher, and Borje Ohlman. A survey of information-centric networking. IEEE Communications Magazine, 50(7), 2012.

[92] Qing-Yi Zhang, Xing-Wei Wang, Min Huang, Ke-Qin Li, and Sajal K Das. Software defined networking meets information centric networking: A survey. IEEE Access, 6:39547-39563, 2018.

Received 0; revised 0; accepted 0 